Department of Mathematics, Statistics and Computer Science

University of Illinois at Chicago

851 South Morgan, Chicago, IL 60607, USA 


\title{
The structure of free algebras
}

\author{
Joel BERMAN
}

\begin{abstract}
This article is a survey of selected results on the structure of free algebraic systems obtained during the past 50 years. The focus is on ways free algebras can be decomposed into simpler components and how the number of components and the way the components interact with each other can be readily determined. A common thread running through the exposition is a concrete method of representing a free algebra as an array of elements.
\end{abstract}

\section{Introduction}

Let $\mathcal{V}$ be a variety (or equational class) of algebras. By $\mathbf{F}_{\mathcal{V}}(X)$ we denote the free algebra for $\mathcal{V}$ freely generated by the set $X$. The algebra $\mathbf{F}_{\mathcal{V}}(X)$ may be defined as an algebra in $\mathcal{V}$ generated by $X$ that has the universal mapping property: For every $\mathbf{A} \in \mathcal{V}$ and every function $v: X \rightarrow A$ there is a homomorphism $h: \mathbf{F}_{\mathcal{V}}(X) \rightarrow \mathbf{A}$ that extends $v$, i.e., $h(x)=v(x)$ for all $x \in X$. It is known that $\mathbf{F}_{\mathcal{V}}(X)$ exists for every variety $\mathcal{V}$ and is unique up to isomorphism.

In this article we investigate the structure of free algebras in varieties. We present a very concrete, almost tactile, representation of $\mathbf{F}_{\mathcal{V}}(X)$ and some algebras closed related to $\mathbf{F}_{\mathcal{V}}(X)$ as a rectangular arrays of elements. The rows of such an array are indexed by the elements of the algebra and the columns are indexed by a set $U$ of valuations, where a valuation is any function $v$ from $X$ to an algebra $\mathbf{A} \in \mathcal{V}$ for which the set $v(X)$ generates all of $\mathbf{A}$. This array is denoted $\mathbf{G e}(X, U)$.

In Section 1 we prove some general results about $\mathbf{G e}(X, U)$. We are interested in finding small tractable sets $U$ of valuations that can be used to represent $\mathbf{F}_{\mathcal{V}}(X)$. Several such $U$ are described. The section also contains a detailed description of how a software package developed by $\mathrm{R}$. Freese and E. Kiss can be used to explicitly construct the array $\mathbf{G e}(X, U)$ for finitely generated varieties $\mathcal{V}$ and finite $X$. The remaining three sections deal with decompositions of $\mathbf{F}_{\mathcal{V}}(X)$ into well-behaved substructures, and how these substructures are organized among themselves in a systematic way. In Section 2 the substructures considered are congruence classes of the kernel of a canonical homomorphism of $\mathbf{F}_{\mathcal{V}}(X)$ onto $\mathbf{F}_{\mathcal{W}}(X)$ for $\mathcal{W}$ a well-behaved and well-understood subvariety of $\mathcal{V}$. In Section 3 varieties $\mathcal{V}$ are described for which $\mathbf{F}_{\mathcal{V}}(X)$ can be decomposed into overlapping syntactically defined substructures that are very homogeneous and code, in various ways, families of finite ordered sets. Because of the way these subsets fit together, an inclusion-exclusion type of argument can be used to determine the cardinality of $\mathbf{F}_{\mathcal{V}}(X)$. The final section deals with direct decompositions of $\mathbf{F}_{\mathcal{V}}(X)$ into a product of directly indecomposable factors. Some specific general conditions on a variety $\mathcal{V}$ are provided that allow for the determination of the structure of the directly indecomposable factors and of the exact multiplicity of each factor in the product. 
For general facts about varieties, free algebras and universal algebra used in this paper the reader may consult [8] or [18]. For the most part we follow the notation and terminology found there.

For a set $X$ of variables and a variety $\mathcal{V}$ of similarity type $\tau$, a typical term of type $\tau$ built from $X$ is denoted $t\left(x_{1}, \ldots, x_{n}\right)$, where the $x_{i}$ are elements of $X$. If $n$ and $X$ are clear from the context we simply write $t$. The set of variables that actually appear in a term $t$ is denoted $\operatorname{var}(t)$. For a term $t\left(x_{1}, \ldots, x_{n}\right)$ and an algebra $\mathbf{A}$ in $\mathcal{V}$, by $t^{\mathbf{A}}$ we denote the the term operation on $\mathbf{A}$ corresponding to $t$. Thus $t^{\mathbf{A}}: A^{n} \rightarrow A$ and for $a_{1}, \ldots, a_{n} \in A$, $t^{\mathbf{A}}\left(a_{1}, \ldots, a_{n}\right)$ is the element of $A$ obtained by interpreting $t$ to the fundamental operations of $\mathbf{A}$ and applying the resulting operation to $a_{1}, \ldots, a_{n}$. The universe of $\mathbf{F}_{\mathcal{V}}(X)$ is denoted $\mathrm{F}_{\mathcal{V}}(X)$ and we use the following notation for elements of $\mathrm{F}_{\mathcal{V}}(X)$ : For $x \in X$ we write $\bar{x}$ for the element of $\mathrm{F}_{\mathcal{V}}(X)$ obtained by applying $x^{\mathbf{F}_{\mathcal{V}}(X)}$ to the generator $x$ of $\mathbf{F}_{\mathcal{V}}(X)$ and for a term $t\left(x_{1}, \ldots, x_{n}\right)$ we write $\bar{t}$ for $t^{\mathbf{F}_{\mathcal{V}}(X)}\left(\bar{x}_{1}, \ldots, \bar{x}_{n}\right)$. Note that $x=\bar{x}$ for every $x \in X$, every element of $\mathbf{F} \mathcal{V}(X)$ is of the form $\bar{t}$ for some term $t$, and that for distinct terms $s$ and $t$ we can have $\bar{s}=\bar{t}$. A crucial fact about free algebras used repeatedly throughout this paper is that $\mathcal{V}$ satisfies the identity $s \approx t$ for terms $s$ and $t$ if and only if the elements $\bar{s}$ and $\bar{t}$ are equal in $\mathrm{F}_{\mathcal{V}}(X)$. Written more succinctly this is the familiar

$$
\mathcal{V} \models s \approx t \quad \text { iff } \quad \bar{s}=\bar{t} .
$$

See, for example $\S 11$ of [8] for further details.

For an algebra $\mathbf{A}$ and a set $X$ we view each element $v$ in the direct power $\mathbf{A}^{X}$ as a function $v: X \rightarrow A$. Given $v \in \mathbf{A}^{X}$ we denote the algebra $\mathbf{A}$ by $\mathbf{A} \lg (v)$. If $v \in \mathbf{A}^{X}$ and $t\left(x_{1}, \ldots, x_{n}\right)$ is a term in the variables of $X$, then $v(t)$ denotes $t^{\mathbf{A}}\left(v\left(x_{1}\right), \ldots, v\left(x_{n}\right)\right)$. We say $v$ is a valuation if $v(X)$ generates the algebra $\mathbf{A} \lg (v)$. The set of all valuations from $X$ to an algebra $\mathbf{A}$ is denoted $\operatorname{val}(X, \mathbf{A})$. For $\mathcal{K}$ a class of algebras, $\operatorname{val}(X, \mathcal{K})$ denotes the collection of all $v \in \operatorname{val}(X, \mathbf{A})$ for $\mathbf{A} \in \mathcal{K}$. Valuations will play a central role in our exposition.

If an algebra $\mathbf{B}$ is the direct product $\prod_{j \in J} \mathbf{B}_{j}$, then we denote by $p r_{j}$ the projection homomorphism from $\mathbf{B}$ onto $\mathbf{B}_{j}$. For $K \subseteq J$ the projection of $\mathbf{B}$ onto $\prod_{j \in K} \mathbf{B}_{j}$ is denoted $\operatorname{pr}_{K}$.

Let $\mathcal{K}$ be a set of algebras of the same similarity type, $X$ a set, and $U$ a subset of $\bigcup\left(\mathbf{A}^{X}: \mathbf{A} \in \mathcal{K}\right)$. For $x \in X$ let $\bar{x} \in \prod_{v \in U} \mathbf{A l g}(v)$ denote the element given by $p r_{v}(\bar{x})=v(x)$ for all $v \in U$. We let $\bar{X}=\{\bar{x}: x \in X\}$. The subalgebra of $\prod_{v \in U} \mathbf{A} \lg (v)$ generated by $\bar{X}$ is denoted $\mathbf{G e}(X, U)$.

1.1 Lemma Let $\mathcal{V}$ be a variety and $U \subseteq \bigcup\left(\mathbf{A}^{X}: \mathbf{A} \in \mathcal{V}\right)$. If $\operatorname{val}(X, \mathcal{V}) \subseteq U$, then $\mathbf{G e}(X, U)$ is isomorphic to $\mathbf{F}_{\mathcal{V}}(X)$.

Proof The algebra $\operatorname{Ge}(X, U)$ is generated by $\bar{X}$. The set $U$ contains valuations to separate the elements of $X$, so $X$ and $\bar{X}$ have the same cardinality. So it suffices to show that $\mathbf{G e}(X, U)$ has the universal mapping property for $\bar{X}$ over $\mathcal{V}$. Let $w$ be any function from $\bar{X}$ into an algebra $\mathbf{A} \in \mathcal{V}$. Let $v \in \operatorname{val}\left(X, \mathrm{Sg}^{\mathbf{A}}(\mathrm{w}(\mathrm{X}))\right)$ be defined by $v(x)=w(\bar{x})$ for every $\bar{x} \in \bar{X}$. By assumption $v \in U$. Then $p r_{v}$ is a homomorphism from $\mathbf{G e}(X, U)$ into $\mathbf{A}$ that extends $w$ since $p r_{v}(\bar{x})=v(x)=w(\bar{x})$.

1.2 Definition For a variety $\mathcal{V}$ and a set $X$, we call $U \subseteq \operatorname{val}(X, \mathcal{V})$ free for $\mathcal{V}$ if $\mathbf{G e}(X, U)$ is isomorphic to $\mathbf{F}_{\mathcal{V}}(X)$. The set $U$ is called independent for $\mathcal{V}$ if $\mathbf{G e}(X, U)$ is isomorphic to $\prod_{v \in U} \mathbf{A} \lg (v)$. 
We are very much interested in concrete representations of $\mathbf{G e}(X, U)$ when $U$ is free for $\mathcal{V}$. To this end we first consider sufficient conditions on a set $U \subseteq \operatorname{val}(X, \mathcal{V})$ that force $U$ to be independent.

1.3 Lemma Let $\mathcal{V}$ be a variety and suppose $U \subseteq \operatorname{val}(X, \mathcal{V})$ is such that for every pair of terms $s$ and $t$ for which $\mathcal{V} \not=s \approx t$ there exists $v \in U$ such that $v(s) \neq v(t)$. Then $\operatorname{Ge}(X, U) \cong$ $\mathbf{F}_{\mathcal{V}}(X)$, that is, $U$ is free for $\mathcal{V}$.

Proof By virtue of the previous lemma it suffices to show that the projection homomorphism $p r_{U}$ from $\mathbf{G e}(X, \operatorname{val}(X, \mathcal{V}))$ onto $\mathbf{G e}(X, U)$ is one-to-one. If $s\left(\bar{x}_{1}, \ldots, \bar{x}_{n}\right)$ and $t\left(\bar{x}_{1}, \ldots, \bar{x}_{n}\right)$ are distinct elements of $\mathbf{G e}(X, \operatorname{val}(X, \mathcal{V}))$, then $\mathcal{V} \not \models s \approx t$. So there exists $v \in U$ for which $v(s) \neq$ $v(t)$. Then $\operatorname{pr}_{v}\left(s\left(\bar{x}_{1}, \ldots, \bar{x}_{n}\right)\right)=s\left(v\left(x_{1}\right), \ldots, v\left(x_{n}\right)\right)=v(s) \neq v(t)=t\left(v\left(x_{1}\right), \ldots, v\left(x_{n}\right)\right)=$ $\operatorname{pr}_{v}\left(s\left(\bar{x}_{1}, \ldots, \bar{x}_{n}\right)\right)$. Thus, $\operatorname{pr}_{U}\left(s\left(\bar{x}_{1}, \ldots, \bar{x}_{n}\right)\right) \neq \operatorname{pr}_{U}\left(t\left(\bar{x}_{1}, \ldots, \bar{x}_{n}\right)\right)$.

1.4 Corollary Let $\mathcal{V}$ be a variety and $X$ a set.

(1) If $\mathcal{V}_{\mathrm{SI}}$ is the class of (finitely generated) subdirectly irreducible algebras in $\mathcal{V}$ and if $U=\operatorname{val}\left(X, V_{\mathrm{SI}}\right)$, then $U$ is free for $\mathcal{V}$.

(2) If $\mathcal{V}$ is generated by the finite algebras $\mathbf{A}_{1}, \ldots, \mathbf{A}_{m}$ and if $U=\bigcup\left(\mathbf{A}_{i}^{X}: 1 \leq i \leq m\right)$, then $U$ is free for $\mathcal{V}$ and $\left|\mathbf{F}_{\mathcal{V}}(n)\right| \leq \prod_{i=1}^{m}\left|A_{i}\right|^{\left|A_{i}\right|^{n}}$.

The second part of this Corollary is presented in Birkhoff's 1935 paper [7].

1.5 Definition Let $\mathcal{V}$ be an arbitrary variety and $X$ a set. Given two valuations $v$ and $v^{\prime}$ with $\mathbf{A}=\mathbf{A} \lg (v)$ and $\mathbf{A}^{\prime}=\mathbf{A} \lg \left(v^{\prime}\right)$ algebras in $\mathcal{V}$, we say that $v$ and $v^{\prime}$ are equivalent, written $v \sim v^{\prime}$, if there exist homomorphisms $h: \mathbf{A} \rightarrow \mathbf{A}^{\prime}$ and $h^{\prime}: \mathbf{A}^{\prime} \rightarrow \mathbf{A}$ such that $v^{\prime}=h v$ and $v=h^{\prime} v^{\prime}$. For any set $U \subseteq \operatorname{val}(X, \mathcal{V})$ let $E(U)$ denote any transversal of $\sim$ over elements of $U$. That is, $E(U)$ is any subset of $U$ that consists of exactly one valuation taken from each equivalence class of $\sim$.

1.6 Lemma If $U$ is any set of valuations, then $\mathbf{G e}(X, U) \cong \mathbf{G e}(X, E(U))$.

Proof The set $E(U)$ is a subset of $U$, and the projection $p r_{e v(U)}: \mathbf{G e}(X, U) \rightarrow \mathbf{G e}(X, E(U))$ is an onto homomorphism. It suffices to show $\operatorname{pr}_{E(U)}$ is one-to-one. Let $\bar{s} \neq \bar{t}$ in $\mathbf{G e}(X, U)$. So there is a $v \in U$ for which $v(s) \neq v(t)$. Let $v^{\prime} \in E(U)$ be such that $v \sim v^{\prime}$. Then there is a homomorphism $h^{\prime}$ for which $v=h^{\prime} v^{\prime}$. If $v^{\prime}(s)=v^{\prime}(t)$, then $v(s)=v(t)$, which is impossible. So $v^{\prime}(s) \neq v^{\prime}(t)$ and hence $\operatorname{pr}_{E(U)}(\bar{s}) \neq p r_{E(U)}(\bar{t})$.

1.7 Corollary If a set $U$ of valuations is free for $\mathcal{V}$, then so is $E(U)$.

Henceforth in this paper, unless otherwise indicated, $X$ will denote the set $\left\{x_{1}, \ldots, x_{n}\right\}$. Under this convention $\mathbf{F}_{\mathcal{V}}(X)$ and $\mathbf{F}_{\mathcal{V}}(n)$ are the same.

A variety $\mathcal{V}$ is locally finite if every finitely generated algebra in $\mathcal{V}$ is finite. For a locally finite variety, if $f(n)$ is the cardinality of $\mathbf{F}_{\mathcal{V}}(n)$, then every at most $n$ generated algebra in $\mathcal{V}$ has cardinality at most $f(n)$. The number of valuations $v$ from a set $X$ of size $n$ to a particular algebra $\mathbf{A}$ is bounded above by $|A|^{n}$, which is at most $f(n)^{n}$. 
Let $\mathcal{V}$ be a locally finite variety, $X=\left\{x_{1}, \ldots, x_{n}\right\}$, and $U \subseteq \operatorname{val}(X, \mathcal{V})$. We present a concrete representation of $\mathbf{G e}(X, U)$ as a rectangular array of elements from $\mathbf{A} \lg (v)$ for $v$ ranging over $U$. Each $v \in U$ determines a column in this array and each element of $\mathbf{G e}(X, U)$ determines a row. The first $n$ rows are indexed by $\bar{x}_{1}, \ldots, \bar{x}_{n}$. If $t\left(x_{1}, \ldots, x_{n}\right)$ is any term, then $\bar{t}$ denotes $t^{\mathbf{G e}(X, U)}\left(\bar{x}_{1}, \ldots, \bar{x}_{n}\right)$. Since the $\bar{x}_{i}$ generate $\mathbf{G e}(X, U)$, every element of $\mathbf{G e}(X, U)$ is of the form $\bar{t}$ for some term $t$. For $v \in U$ and $\bar{t}=t\left(\bar{x}_{1}, \ldots, \bar{x}_{n}\right) \in \mathbf{G e}(X, U)$, the entry in row $\bar{t}$ and column $v$ is $v(t) \in \mathbf{A} \lg (v)$. Note that $v(t)=t^{\mathbf{A l g}(v)}\left(v\left(x_{1}\right), \ldots, v\left(x_{n}\right)\right)$. In column $v$, every element of $\mathbf{A} \lg (v)$ appears at least once since $v$ is a valuation. Moreover, column $v$ codes the projection $p r_{v}$ of $\mathbf{G e}(X, U)$ onto the algebra $\mathbf{A} \lg (v)$.

In the special case that $\mathcal{V}$ is generated by a finite algebra $\mathbf{A}$ and $U=\mathbf{A}^{X}$, then $U$ is free for $\mathcal{V}$ by Corollary 1.4, the number of columns of $\mathbf{G e}(X, U)$ is $|A|^{n}$, and the number of rows of $\mathbf{G e}(X, U)$ is $\left|\mathrm{F}_{\mathcal{V}}(n)\right|$.

We next present three examples of how $\mathbf{G e}(X, U)$ may be used to describe the structure of free algebras.

1.8 Example Let $\mathcal{S}$ be the variety of semilattices. We show how to find a normal form for an arbitrary term and how to use this normal form to determine the structure of free semilattices. If $t\left(x_{1}, \ldots, x_{n}\right)$ is any semilattice term, then by associativity we may ignore the parentheses and write $t$ as a string of variables. By commutativity we may sort the variables in the string by increasing subscripts. Idempotence allows us to conclude $\mathcal{S} \models t \approx x_{i_{1}} x_{i_{2}} \ldots x_{i_{k}}$ where $1 \leq i_{1}<i_{2}<\cdots<i_{k} \leq n$ and $\operatorname{var}(t)=\left\{x_{i_{1}}, x_{i_{2}}, \ldots, x_{i_{k}}\right\}$. Thus a concrete representation of $\mathbf{F}_{\mathcal{S}}(X)$ as a join semilattice is the set of nonvoid subsets of $X$ with the operation of union. In particular $\left|\mathrm{F}_{\mathcal{S}}(X)\right|=2^{n}-1$.

The variety $\mathcal{S}$ is generated by the 2 -element semilattice $\mathbf{S}_{2}$ with universe $\{0,1\}$. Without loss of generality $\mathbf{S}_{2}$ is a join semilattice. The set $\operatorname{val}\left(X, \mathbf{S}_{2}\right)$ has $2^{n}-2$ elements. Thus, $\mathbf{F}_{\mathcal{S}}(X)$ is isomorphic to $\mathbf{G e}\left(X, \operatorname{val}\left(X, \mathbf{S}_{2}\right)\right)$. If we view $\mathbf{G e}\left(X, \operatorname{val}\left(X, \mathbf{S}_{2}\right)\right)$ as an array, then it has $2^{n}-2$ columns and $2^{n}-1$ rows. If $U \subseteq \operatorname{val}\left(X, \mathbf{S}_{2}\right)$ consists of the valuations $v_{i}$ for $1 \leq i \leq n$ where $v_{i}\left(x_{j}\right)=1$ if and only if $i=j$, then $U$ is free for $\mathcal{S}$. To see this, it suffices to show that the projection $p r_{U}$ is one-to-one. Suppose $\bar{t}=t\left(\bar{x}_{1}, \ldots, \bar{x}_{n}\right)$ and $\bar{s}=s\left(\bar{x}_{1}, \ldots, \bar{x}_{n}\right)$ are distinct elements of $\mathbf{G e}\left(X, \operatorname{val}\left(X, \mathbf{S}_{2}\right)\right)$. Let $v$ be any valuation into $\mathbf{S}_{2}$ that separates $\bar{s}$ and $\bar{t}$, with say $v(t)=1$. So there exists $x_{i} \in \operatorname{var}(t)-\operatorname{var}(s)$. Then $v_{i}\left(x_{i}\right)=1=v_{i}(t)$ while $v_{i}(s)=0$. So a valuation $v_{i}$ in $U$ separates $\bar{t}$ and $\bar{s}$. Hence $U$ is free for $\mathcal{S}$. The array for $\operatorname{Ge}(X, U)$ has $n$ columns and $2^{n}-1$ rows. A cardinality argument shows that no proper subset of $U$ is free for $\mathcal{S}$. Although $U$ is free for $\mathbf{S}$, it is not independent since there is no $\bar{t}$ having $v(t)=0$ for all $v$. However, if $\mathcal{S}$ were the variety of join semilattices with constant 0 , then $U$ would be both free and independent for $\mathcal{S}$ with $\mathbf{F}_{\mathcal{S}}(n) \cong \mathbf{S}_{2}^{n}$ for $\mathbf{S}_{2}$ the 2-element join semilattice with constant 0 .

1.9 Example Let $\mathcal{B}$ be the variety of Boolean algebras. This variety is generated by the 2element Boolean algebra $\mathbf{B}_{2}=\left\langle\{0,1\}, \wedge, \vee,{ }^{\prime}, 0,1\right\rangle$, which is the only subdirectly irreducible algebra in the variety. Note that $\operatorname{val}\left(X, \mathbf{B}_{2}\right)=\mathbf{B}_{2}^{X}$. For $v \in \operatorname{val}\left(X, \mathbf{B}_{2}\right)$ let $t_{v}$ be the term $x_{1}^{v\left(x_{1}\right)} \wedge \cdots \wedge x_{n}^{v\left(x_{n}\right)}$, where $x_{i}^{0}=x_{i}^{\prime}$ and $x_{i}^{1}=x_{i}$. Then $v\left(t_{v}\right)=1$ but $w\left(t_{v}\right)=0$ for every valuation $w \neq v$. The array for $\mathbf{G e}\left(X, \operatorname{val}\left(X, \mathbf{B}_{2}\right)\right)$ has $2^{n}$ columns. By considering joins of the $2^{n}$ different $t_{v}$ we see that there are $2^{2^{n}}$ rows. So $\operatorname{val}\left(X, \mathbf{B}_{2}\right)$ is free for $\mathcal{B}$ and is independent but has no proper subset that is free for $\mathcal{B}$. Thus $\mathbf{F}_{\mathcal{B}}(n) \cong \mathbf{B}_{2}^{n}$. The $t_{v}$ are sometimes called minterms. The array for $\mathbf{G e}\left(X, \operatorname{val}\left(X, \mathbf{B}_{2}\right)\right)$ may be viewed as the collection of all truth 
tables for Boolean terms involving the variables from $X$. The only difference being that in truth tables the result of applying all valuations to a given term is given as a column vector whereas in the array for $\operatorname{val}\left(X, \mathbf{B}_{2}\right)$ this is presented as a row vector.

1.10 Example Let $\mathbf{C}_{2}$ be the 2-element implication algebra $\langle\{0,1\},, \rightarrow\rangle$ and $\mathcal{I}$ the variety it generates. In $\mathbf{C}_{2}$ we have $1 \rightarrow 0=0$ and $a \rightarrow b=1$ otherwise. Algebras in $\mathcal{I}$ have an order defined on them by $x \leq y$ if and only if $x \rightarrow y=1$. The term operation $\left(x_{1} \rightarrow x_{2}\right) \rightarrow x_{2}$ is the join operation for this order. We consider $\mathbf{G e}\left(X, \mathbf{C}_{2}^{X}\right)$, which is isomorphic to $\mathbf{F}_{\mathcal{I}}(X)$. For $\bar{t} \in \mathbf{G e}\left(X, \mathbf{C}_{2}^{X}\right)$ we have $\bar{x}_{i} \leq \bar{t}$ if and only if $v\left(x_{i}\right) \leq v(t)$ for all $v \in \mathbf{C}_{2}^{X}$. For $1 \leq i \leq n$ let $\bar{T}_{i}=\left\{\bar{t} \in \mathbf{G e}\left(X, \mathbf{C}_{2}^{X}\right): \bar{x}_{i} \leq \bar{t}\right\}$. Then $\bar{T}_{i}$ is a subuniverse of $\mathbf{G e}\left(X, \mathbf{C}_{2}^{X}\right)$ since $y \leq x \rightarrow y$ holds for all elements in algebras in $\mathcal{I}$. Moreover, every $\bar{t}$ is in at least one $\bar{T}_{i}$. Let $U_{i}$ consist of all $v \in \mathbf{C}_{2}^{X}$ for which $v\left(x_{i}\right)=0$. The array for $\mathbf{G e}\left(X, U_{i}\right)$ has $2^{n-1}$ columns. For $\bar{t} \in \mathbf{G e}\left(X, U_{i}\right)$ and $v \in U_{i}$ we have that $v\left(t \rightarrow x_{i}\right)=t^{\prime}$ for ' the complementation operation on $\mathbf{B}_{2}$. Thus, on $\mathbf{G e}\left(X, U_{i}\right)$ we have term operations that are the Boolean operations $\vee$ and '. The element $\bar{x}_{i}$ serves as the Boolean 0 here. So $\mathbf{G e}\left(X, U_{i}\right)$ contains all Boolean term operations that can be generated by the $n-1$ elements $\bar{x}_{1}, \ldots, \bar{x}_{i-1}, \bar{x}_{i+1}, \ldots, \bar{x}_{n}$. Thus $\bar{T}_{i}$ contains $2^{2^{n-1}}$ elements. If $\overline{\mathbf{T}}_{i}$ is the algebra with universe $\bar{T}_{i}$, then $\overline{\mathbf{T}}_{i}$ and $\left(\mathbf{C}_{2}\right)^{2^{n-1}}$ are isomorphic. From the facts that $\mathbf{G e}\left(X, \mathbf{C}_{2}^{X}\right)=\bar{T}_{1} \cup \cdots \cup \bar{T}_{n}$, all the $\overline{\mathbf{T}}_{i}$ are isomorphic, and $\left|\bar{T}_{1} \cap \cdots \cap \bar{T}_{k}\right|=2^{2^{n-k}}$ for every $1 \leq k \leq n$, it follows from a standard inclusion-exclusion argument that

$$
\left|\mathbf{F}_{\mathcal{I}}(n)\right|=\left|\mathbf{G e}\left(X, \mathbf{C}_{2}^{X}\right)\right|=\sum_{k=1}^{n}(-1)^{k}\left(\begin{array}{l}
n \\
k
\end{array}\right) 2^{2^{n-k}} .
$$

In the previous examples we considered a finite algebra $\mathbf{A}$ that generates a variety $\mathcal{V}$ and we determined the free algebra $\mathbf{F}_{\mathcal{V}}(X)$ by means of an analysis of the array $\mathbf{G e}(X, U)$ for some $U \subset \mathbf{A}^{X}$ that is free for $\mathcal{V}$. The algebras $\mathbf{A}$ considered have a 2-element universe and a particularly transparent structure. For more complicated finite algebras $\mathbf{A}$, it is usually more difficult to analyze $\mathbf{G e}(X, U)$. However, the array for $\mathbf{G e}(X, U)$ can, in principle, be computed mechanically since it is a subalgebra of $\mathbf{A}^{U}$ generated by the row vectors $\bar{x}_{1}, \ldots, \bar{x}_{n}$. The natural algorithm for finding a subuniverse of an algebra generated by a given generating set can be implemented as a computer program.

Emil W. Kiss and Ralph Freese have developed a software package for doing computations in universal algebra. The package is the Universal Algebra Calculator (UAC) and is freely available from either author's webpage [11]. The menu of programs allows the user to compute all congruence relations of an algebra, view and manipulate the congruence lattice, find factor algebras for a given congruence relation, and answer questions about the algebra that arise from commutator theory and tame congruence theory. Two programs in the package are extremely useful for work on the structure of free algebras. One is a program that with the input of a finite algebra $\mathbf{A}$ and positive integer $n$, determines the free algebra $\mathbf{F}_{\mathcal{V}}(n)$ for $\mathcal{V}$ the variety generated by $\mathbf{A}$. The second takes as input a finite collection of finite algebras $\mathbf{A}_{1}, \ldots, \mathbf{A}_{m}$ of the same similarity type and a set of vectors $\bar{z}_{1}, \ldots, \bar{z}_{n}$, with each $\bar{z}_{i} \in \mathbf{A}_{1} \times \cdots \times \mathbf{A}_{m}$, and produces as output the subalgebra of $\mathbf{A}_{1} \times \cdots \times \mathbf{A}_{m}$ generated by $\bar{z}_{1}, \ldots, \bar{z}_{n}$. This program can of course be used to compute $\mathbf{G e}(X, U)$. These programs are especially useful for analyzing examples and for conducting computer experiments on specific algebras. We next present a detailed example of how the Universal Algebra Calculator might be used in this experimental manner to investigate free algebras in a finitely generated variety. 
1.11 Example For every finite poset $\langle P, \leq\rangle$ with a top element 1 define a finite algebra $\mathbf{P}=\langle P, \cdot\rangle$ by

$$
x \cdot y= \begin{cases}1 & \text { if } x \leq y \\ y & \text { otherwise }\end{cases}
$$

Let $\mathcal{V}$ be the variety generated by all such $\mathbf{P}$. The general problem is to determine the structure of $\mathbf{F}_{\mathcal{V}}(n)$ for all positive $n$. A more specific problem is to determine the free algebras in a variety generated by a single algebra $\mathbf{P} \in \mathcal{V}$.

If $\mathbf{A}$ is any algebra in $\mathcal{V}$, then it is not hard to show that the binary relation $\leq$ on $A$ given by $x \leq y$ if and only if $x \cdot y=1$ is an order relation. Note that although 1 is not in the similarity type of $\mathcal{V}$, the term $x \cdot x$ will serve in its place.

If $\mathbf{P}$ is the algebra arising from the two element chain $0<1$, then $\mathbf{P}=\mathbf{C}_{2}$ as in Example 1.10, and we have described there the structure of the free algebras in the variety generated by $\mathbf{C}_{2}$. So let us next consider the algebra arising from the 3-element chain $2<0<1$. If $\mathbf{C}_{3}$ denotes this algebra, then the operation $x \cdot y$ on $\mathbf{C}_{3}$ is given by the following table.

\begin{tabular}{|l||l|l|l|}
\hline$\cdot$ & 0 & 1 & 2 \\
\hline \hline 0 & 1 & 1 & 2 \\
\hline 1 & 0 & 1 & 2 \\
\hline 2 & 1 & 1 & 1 \\
\hline
\end{tabular}

We can use this table as input for the Universal Algebra Calculator. The UAC can then calculate the free algebra on two free generators for the variety $\mathcal{V}$ generated by this input algebra. A tabular printout for the result of this computation is given in Table 1.

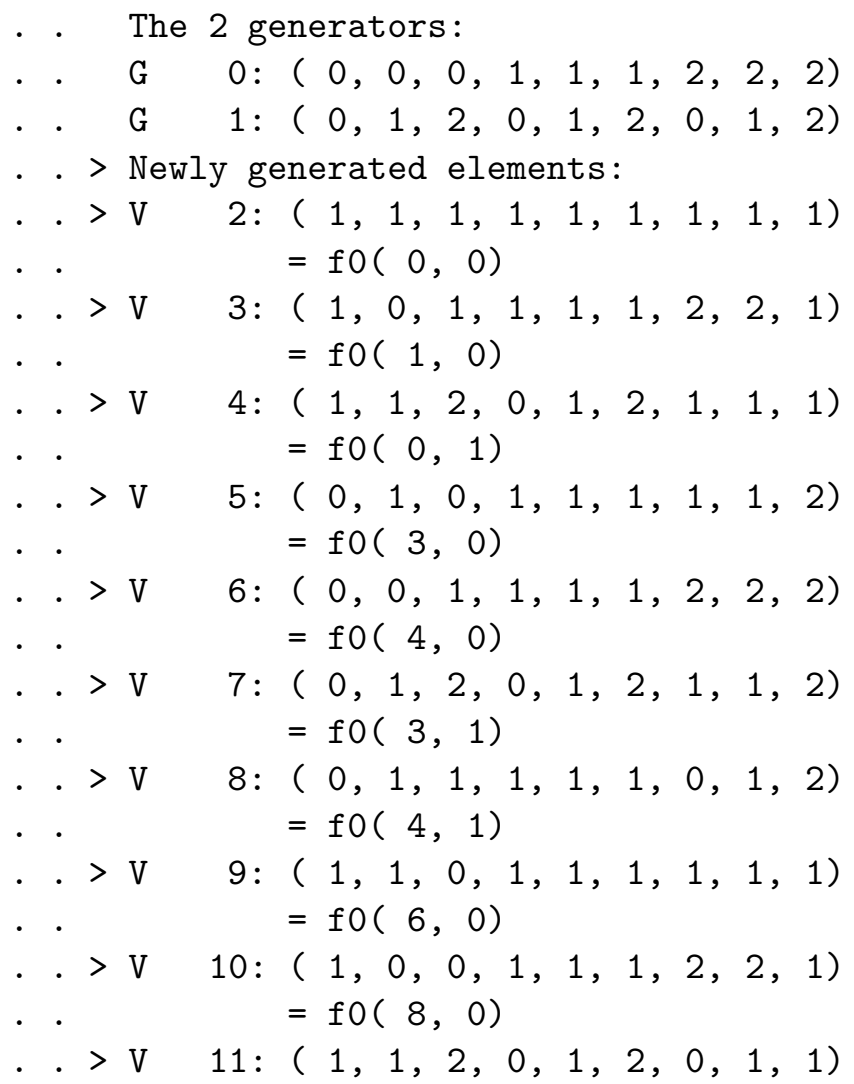




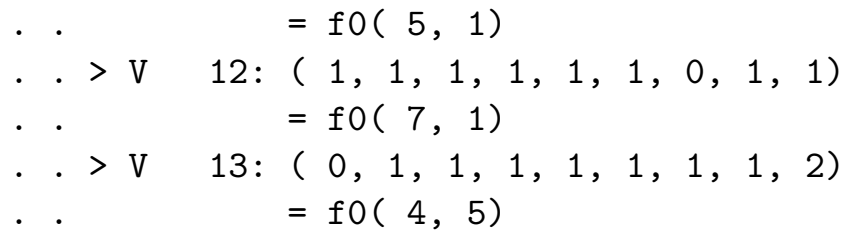

Table 1

The algebra $\mathbf{F}_{\mathcal{V}}(2)$ is represented as a subset of $\left(\mathbf{C}_{3}\right)^{3^{2}}$. The elements are represented as 9 -tuples with the two generators having the label $\mathrm{G}$ and numbered 0 and 1 . The other elements in the free algebra are labeled with the letter $\mathrm{V}$ and are numbered 2 through 13. The binary operation on the algebra is denoted $\mathbf{f} 0$. Information about how each generated element is obtained is explicitly given. Thus, in the last two lines of the table we see that element 13 is obtained by applying $\mathrm{f} 0$ to elements 4 and 5 . A printout of this data in a more streamlined form is given in Table 2. Here we see a representation of $\mathbf{F}_{\mathcal{V}}(2)$ as we view the array $\mathbf{G e}(X, U)$ for $X$ the two generating elements and $U$ the 9 functions $\{0,1,2\}^{X}$. This illustrates how the UAC program gives a concrete representation of the array $\operatorname{Ge}(X, U)$ for an algebra $\mathbf{A}$ and a set $U \subseteq \mathbf{A}^{X}$.

[ Creating an algebra in a subproduct.

. [ Reading vectorlist file 'C: \algebras\join3f2.uni'.

. . < Number of vectors: 14

. . Length of vectors: 9

. V $\quad 0:(0,0,0,1,1,1,2,2,2)$

. V $\mathrm{V} \quad 1:(0,1,2,0,1,2,0,1,2)$

. V $\mathrm{V} \quad 2:(1,1,1,1,1,1,1,1,1)$

. . $\mathrm{V} \quad 3:(1,0,1,1,1,1,2,2,1)$

. V $\mathrm{V} \quad 4:(1,1,2,0,1,2,1,1,1)$

. V $\quad \mathrm{V}:(0,1,0,1,1,1,1,1,2)$

.. $\mathrm{V} \quad 6:(0,0,1,1,1,1,2,2,2)$

. V V 7: $(0,1,2,0,1,2,1,1,2)$

. V $\mathrm{V}$ 8: $(0,1,1,1,1,1,0,1,2)$

. V $\mathrm{V} \quad$ : $(1,1,0,1,1,1,1,1,1)$

. V $\mathrm{V}$ 10: ( $1,0,0,1,1,1,2,2,1)$

. V $\mathrm{V}$ 11: $(1,1,2,0,1,2,0,1,1)$

. $\mathrm{V}$ 12: $(1,1,1,1,1,1,0,1,1)$

. V V 13: ( $0,1,1,1,1,1,1,1,2)$

. ] End of reading vectors.

- [ Reading algebra 'C: \algebras\join3.alg'.

Table 2

A first approach to understanding the structure of $\mathbf{F}_{\mathcal{V}}(2)$ would be to draw the order relation on the 14 elements of the algebra. This can be done using Table 2 by ordering the vectors coordinatewise by $0<1$. The diagram shows that the two generators are the only minimal elements of this order, that is, every element is greater than or equal to at least one 
generator. This is reminiscent of the situation for the free algebras in the variety $\mathcal{I}$ generated by $\mathbf{C}_{2}$ described in Example 1.10. The entire ordered set is quite complicated, but if we look a single generator and all of the elements greater than or equal to it, we get the ordered set drawn in Figure 1. This ordered set is $\mathbf{C}_{2}{ }^{3}$ with two additional elements labelled 8 and 12 . Note that in $\mathbf{F}_{\mathcal{I}}(2)$ the ordered set of elements above either generator is order isomorphic to $\mathbf{C}_{2}^{3}$. So a natural question to ask is how do 8 and 12 differ from the other eight elements that are above the generator 0 ?

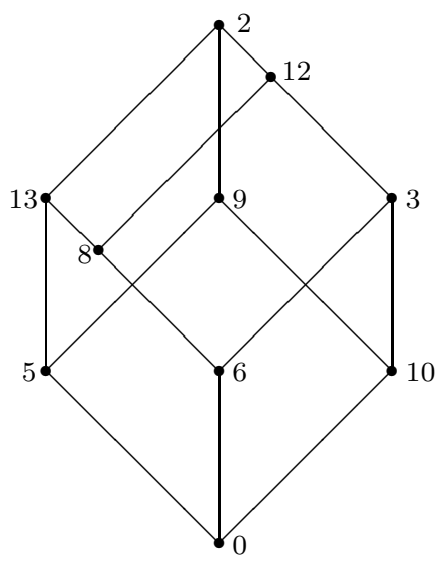

Figure 1

By means of Table 1 we can represent each element in terms of the two generators 0 and 1. The element 8 is (01)1 and the element 12 is ((10)1)1. The other elements in Figure 1 all have the property that each of them can be written as a term involving the generators 0 and 1 with 0 being the rightmost variable that appears. Thus $13=(01)((01) 0)$. The elements 8 and 12 , at least in the representation given by Table 1 , have rightmost variable 1 . One can argue that in any representation of 8 and 12 as a term operation $t$ applied to 0 and 1 the rightmost variable of $t$ will be 1 . This can be done by observing that if $v$ is any valuation of $X$ into $\mathbf{C}_{3}$ and $t\left(x_{1}, \ldots, x_{n}\right)$ is any term with rightmost variable $x_{i}$, then $v(t)=v\left(x_{i}\right)$ or $v(t)=1$.

For the valuation $v$ that sends generator 0 to 2 and generator 1 to 0 , an examination of the appropriate column in Table 1 shows that $v(8)=0$. So 0 could not be the rightmost variable for any representation of element 8. A similar argument works for element 12. This analysis suggests that for a given generator $x_{i}$, rather than look at the set of elements that are greater than or equal to $x_{i}$, we look at those elements that can be written in some way with a term having $x_{i}$ as its rightmost variable. There are $8=2^{3}$ elements representable with terms having 0 as the rightmost variable, and by symmetry there are 8 elements having 1 as rightmost variable, and two elements, 2 and 13, that can be written with either variable as a rightmost variable. We have $14=8+8-2$ and appear to have a decomposition of $\mathbf{F}_{\mathcal{V}}(2)$ into well-structured overlapping blocks.

We next want to find a small set of valuations that can be used to separate those elements of $\mathbf{F}_{\mathcal{V}}(n)$ that have a representation by means of a term with rightmost variable $x_{i}$. In the 
case of $\mathbf{F}_{\mathcal{I}}(n)$ we considered only those valuations $v$ that have $v\left(x_{i}\right)=0$. Could this same condition work for the variety $\mathcal{V}$ ? We experiment with $\mathbf{F}_{\mathcal{V}}(2)$. There are three valuations with $v\left(x_{1}\right)=0$. Let $U$ be this set of valuations and consider $\mathbf{G e}\left(\left\{x_{1}, x_{2}\right\}, U\right)$. The UAC program allows us to generate a subalgebra of product by inputting the algebra, the number of coordinates, and the generating vectors. Thus we can generate the array corresponding to $\operatorname{Ge}\left(\left\{x_{1}, x_{2}\right\}, U\right)$. This is given in Table 3 We see that there are 10 elements generated. Those vectors that contain a 2 cannot be written as a term with rightmost variable $x_{1}$. This leaves $8=2^{3}$ vectors that contain only 0 and 1 . For any such vector $v$ we have $(v \cdot 0) \cdot 0=v$, so all $2^{3}$ of these vectors can be represented by terms in which the rightmost variable is $x_{1}$. So the three valuations in $U$ serve to separate those elements that can be represented by terms with rightmost variable $x_{1}$, and the number of such elements is $\{0,1\}|U|$.

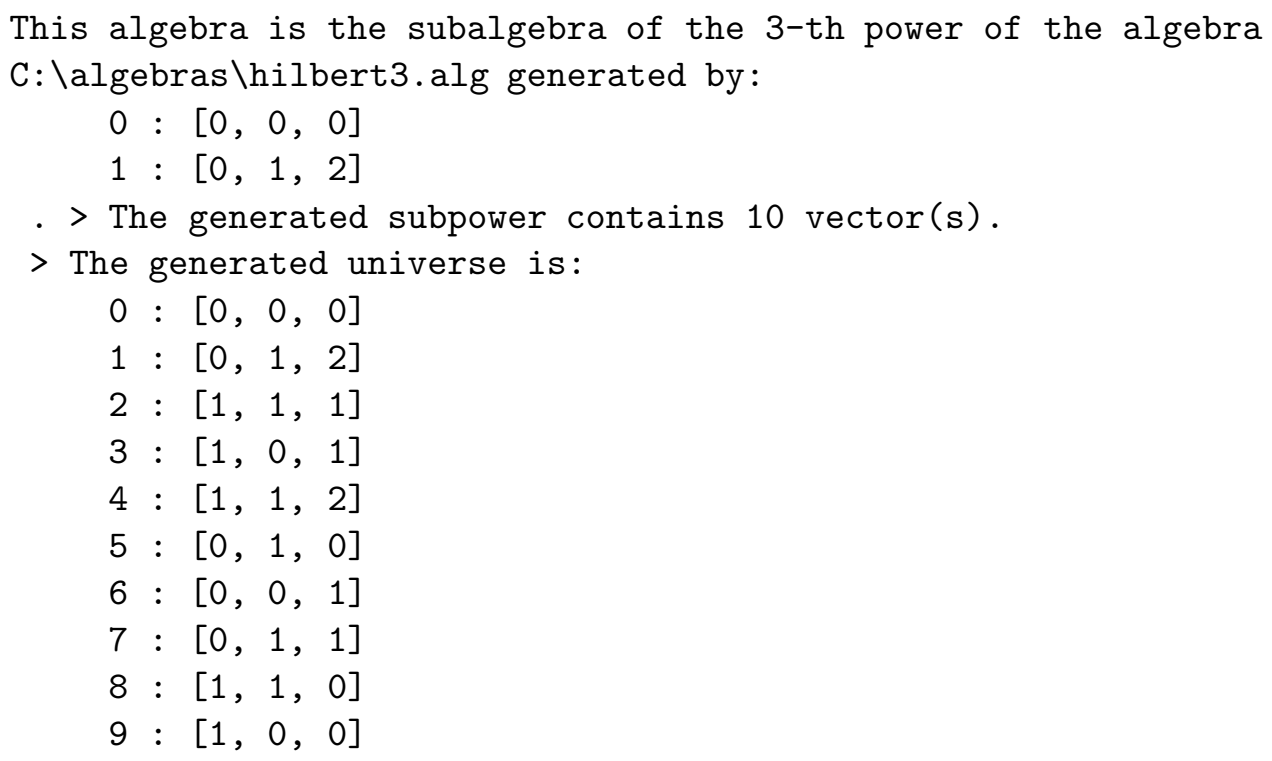

Table 3

On the basis of these computer experiments with $\mathbf{F}_{\mathcal{V}}(2)$ we form this conjecture: For $\mathbf{F}_{\mathcal{V}}(n)$ the subalgebra $\mathbf{T}_{i}$, whose universe consists of those elements that can be written using a term having rightmost variable $x_{i}$, is isomorphic to $\mathbf{C}_{2}^{m}$, where $m$ is the number of $v \in \mathbf{C}_{3}^{X}$ for which $v\left(x_{i}\right)=0$. Thus $\mathbf{T}_{i} \cong\left(\mathbf{C}_{2}\right)^{2^{n-1}}$. If this conjecture were true, then an inclusionexclusion argument as used for the variety $\mathcal{V}$ of implication algebras could be used to find the cardinality of $\mathbf{F}_{\mathcal{V}}(n)$.

We can use the UAC software to check the conjecture for $n=3$. If we just run the program we discover that $\mathbf{F}_{\mathcal{V}}(3)$ has 1514 elements. The algebra is too big, however, to carry out the analysis as we did for $n=2$. We can compute with the program the array corresponding to $\mathbf{G e}(X, U)$ for $X=\left\{x_{1}, x_{2}, x_{3}\right\}$ and $U$ consisting of all $v \in \mathbf{C}_{3}^{X}$ with $v\left(x_{1}\right)=0$. Note that $|U|=9$. The computation shows that there are 558 rows in the array $\operatorname{Ge}(X, U)$. An examination shows that 46 of these rows contain the element 2, which leaves 512 rows in $\{0,1\}^{9}$. Thus, there are at least $2^{9}$ elements of $\mathbf{F}_{\mathcal{V}}(3)$ in $T_{1}$. Likewise if we let $U$ be those $v$ for which $v\left(x_{1}\right)=0$ and $v\left(x_{2}\right)=0$, then we see that there are at least 8 elements in $T_{1} \cap T_{2}$. We also compute that $T_{1} \cap T_{2} \cap T_{3}$ has at cardinality at least 2. By symmetry 
we have $\left|T_{1}\right|=\left|T_{2}\right|=\left|T_{3}\right|$. An inclusion-exclusion argument shows that we have counted $3 * 2^{9}-3 * 2^{3}+2^{1}=1514$ elements. Since 1514 is the cardinality of $\mathbf{F}_{\mathcal{V}}(3)$, we see that $T_{i}$ does indeed have $2^{3^{2}}$ elements, as predicted by the conjecture. So we have used the UAC software to verify the conjecture for $n=3$. In Section 3 we will give a proof of the conjecture in a more general context. The proof will essentially be a formalization of the analysis that we used in our computer experiments in this example.

\section{Structure via decomposition}

We wish to understand the structure of free algebras in a variety. For some varieties the free algebras have a fairly transparent structure. For example, the structure of free semilattices is reasonably clear and is presented in Example 1.8. But for most varieties the structure of the free algebras is too complex to discern in a single view. In some cases one can decompose the free algebras into manageable blocks, and describe how the blocks fit together. If the structure of the blocks is clear and if the manner in which the blocks are related to one another is understood, then a good analysis of the structure of the free algebras in the variety may be provided. In this section we consider a standard and useful method for such decompositions of free algebras. The basic situation is that we are interested in the free algebras in a variety $\mathcal{V}$ and we know that $\mathcal{V}$ has a subvariety $\mathcal{W}$ for which we have some understanding of the algebras $\mathbf{F}_{\mathcal{W}}(n)$. Let $g: \mathbf{F}_{\mathcal{V}}(n) \rightarrow \mathbf{F}_{\mathcal{W}}(n)$ be the canonical map that sends each generator $x_{i}$ to $x_{i}$. Then the kernel of $g$ is a congruence on $\mathbf{F}_{\mathcal{V}}(n)$ whose congruence classes partition $\mathbf{F}_{\mathcal{V}}(n)$. We want $\mathcal{V}$ and $\mathcal{W}$ in which the structure of $\mathbf{F}_{\mathcal{W}}(n)$ and information about the individual congruence classes of the kernel of $g$ may be used to describe $\mathbf{F}_{\mathcal{V}}(n)$. We present several concrete examples of this approach.

2.1 Example The analysis of the free bands by J. A. Green and D. Rees [13] is an excellent example of this method. Let $\mathcal{V}$ be the variety of bands, that is, the variety of semigroups given by the idempotent law $x^{2} \approx x$. The variety $\mathcal{S}$ of semilattices, which is the class of commutative bands, is a subvariety of $\mathcal{V}$. Let $\gamma$ be the kernel of the canonical map $g: \mathbf{F}_{\mathcal{V}}(n) \rightarrow \mathbf{F}_{\mathcal{S}}(n)$ in which $g\left(x_{i}\right)=x_{i}$ for $1 \leq i \leq n$. Recall that for any term $t$, we let $\operatorname{var}(t)$ denote the set of variables that appear in $t$. It is easily seen that for $\bar{s} \in \mathrm{F}_{\mathcal{S}}(n)$ the congruence class $g^{-1}(\bar{s})$ consists those $\bar{t} \in \mathrm{F}_{\mathcal{V}}(n)$ for which $\operatorname{var}(t)=\operatorname{var}(s)$. Moreover, $g^{-1}(\bar{s})$ is a subuniverse of $\mathbf{F}_{\mathcal{V}}(n)$. It can be argued that for $\bar{a}, \bar{b}$ and $\bar{c}$ in $\mathbf{F}_{\mathcal{V}}(n)$, if $(\bar{a}, \bar{c}) \in \gamma$ and $\operatorname{var}(b) \subseteq \operatorname{var}(a)=\operatorname{var}(c)$, then $\bar{a} \bar{b} \bar{c}$ and $\overline{a c}$ are in the same $\gamma$ class. This means that each $\gamma$ class is a rectangular band, that is, the elements in the class satisfy the identity $x y x \approx x$. Thus, the classes of $\gamma$ partition $\mathbf{F}_{\mathcal{V}}(n)$ into rectangular bands and these classes interact together as elements of the free semilattice on $n$ generators. Further structure of the $\gamma$ classes is presented in [13]. It is shown that if $\bar{t} \in \mathbf{F}_{\mathcal{V}}(n)$ with $|\operatorname{var}(t)|=i$, then

$$
|\bar{t} / \gamma|=\prod_{j=1}^{i}(i-j+1)^{2^{j}},
$$

and hence the variety of bands is locally finite with

$$
\left|\mathrm{F}_{\mathcal{V}}(n)\right|=\sum_{i=1}^{n}\left(\begin{array}{l}
n \\
i
\end{array}\right) \prod_{j=1}^{i}(i-j+1)^{2^{j}}
$$


In this type of decomposition in which $\mathbf{F}_{\mathcal{V}}(n)$ is partitioned by a canonical map onto $\mathbf{F}_{\mathcal{W}}(n)$, it is critical that the structure of $\mathbf{F}_{\mathcal{W}}(n)$ be well understood. In the band example the map is onto a free semilattice, which as we have seen has a very transparent structure. Free finitely generated Boolean algebras are also well understood and so for many of the varieties of algebras arising in algebraic logic that contain Boolean algebras as a subvariety, this method of describing the structure of free algebras has been used with some success.

The method may also be used for locally finite varieties of lattices. If $\mathcal{V}$ is any nontrivial variety of lattices, then the variety $\mathcal{D}$ of distributive lattices is a subvariety of $\mathcal{V}$. The free distributive lattice has been the object of intensive study and its structure is understood, although not as thoroughly as that of free semilattices or free Boolean algebras. Thus, an analysis of the partition of $\mathbf{F}_{\mathcal{V}}(n)$ induced by the kernel of the canonical map $g: \mathbf{F}_{\mathcal{V}}(n) \rightarrow$ $\mathbf{F}_{\mathcal{D}}(n)$ might reveal the structure of this free lattice.

We first discuss some the known structure of the free distributive lattice $\mathbf{F}_{\mathcal{D}}(X)$ for $X=\left\{x_{1}, \ldots, x_{n}\right\}$. There is a normal form for distributive lattice terms, as every element can be written as $\bigvee_{i}\left(\bigwedge X_{i}\right)$ where the $X_{i}$ are pairwise incomparable subsets of $X$. This is, of course, the well-known conjunctive normal form. The lattice $\mathbf{F}_{\mathcal{D}}(X)$ has a concrete representation as the lattice of down-sets in the ordered set consisting of all proper nonvoid subsets of $X$. An equivalent representation is the set of proper nonvoid anti-chains in the ordered set $\{0,1\}^{X}$. Yet another representation is as the set of all $n$-ary operations on $\{0,1\}$ that preserve the order relation $0<1$. The ordered set of join-irreducible elements of $\mathbf{F}_{\mathcal{D}}(X)$ is order isomorphic to the set of proper nonvoid subsets of $X$ ordered by inclusion.

Despite all this structural information the problem of actually determining the size of $\mathbf{F}_{\mathcal{D}}(n)$ remains a difficult one. An 1897 paper of Dedekind gives $\left|\mathrm{F}_{\mathcal{D}}(4)\right|=166$. In the years that have followed the cardinalities of free distributive generators on $n$ generators have determined, but with the time interval between successive values of $n$ being 15-20 years. Thus, the exact values of $\mathrm{F}_{\mathcal{V}}(n)$ are known only for $n \leq 8$, with value for $n=8$ being a 23 decimal digit number found in 1991. This suggests that unlike the case for bands, for lattice varieties the decomposition of $\mathbf{F}_{\mathcal{V}}(n)$ into congruence classes of the canonical map onto $\mathbf{F}_{\mathcal{D}}(n)$ will not provide a usable general formula for $\left|\mathrm{F}_{\mathcal{V}}(n)\right|$ as a function of $n$.

Nonetheless, the method has been used to determine the structure and cardinality of free lattices on a small number of generators in some varieties of lattices. The next paragraphs present some of the results from [6] where such an analysis has been performed.

Let $\mathcal{V}$ be any variety of lattices and let $g: \mathbf{F}_{\mathcal{V}}(n) \rightarrow \mathbf{F}_{\mathcal{D}}(n)$ be the homomorphism in which $g\left(x_{i}\right)=x_{i}$ for all variables $x_{i} \in X$. The kernel of $g$ is the congruence relation $\gamma$. For any lattice term $p$ we wish to describe the class $\bar{p} / \gamma$. It can be argued that $g$ is a bounded homomorphism in the sense that the interval $\bar{p} / \gamma$ has a top and bottom element. An argument in [6] shows that if $p_{*}$ is the disjunctive normal form of $p$ and $p^{*}$ is the conjunctive normal form, then $\bar{p} / \gamma$ is the interval $\left[\overline{p_{*}}, \overline{p^{*}}\right]$. Let $U$ be all those valuations $v \in \mathbf{A}^{X}$, as $\mathbf{A}$ ranges of the subdirectly irreducible algebras in $\mathcal{V}$, with $v\left(p_{*}\right) \neq v\left(p^{*}\right)$. Then the interval $\bar{p} / \gamma$ is lattice isomorphic to a sublattice of $\prod_{v \in U} \mathbf{A} \lg (v)$ with the embedding given by $p r_{v}(\bar{q})=v(q)$ for every $\bar{q} \in \bar{p} / \gamma$. The structure of $\bar{p} / \gamma$ is analyzed by determining the collection of joinirreducible elements in this interval lattice. Note that in any finite lattice $L$, the set $J(L)$ of join-irreducible elements generates all of $L$. The following result of $\mathrm{R}$. Wille [22] is useful here.

Let the finite lattice $L$ be a subdirect product of the lattices $L_{i}(i \in I)$. Then $J(L)=$ $\bigcup_{i \in I}\left\{\bigwedge p r_{i}^{-1}(c): c \in J\left(L_{i}\right)\right\}$. 
With this result and the fact that $\bar{p} / \gamma$ is the interval $\left[\overline{p_{*}}, \overline{p^{*}}\right]$, one can, in principle determine the structure of each congruence class $\bar{p} / \gamma$ if one knows $\bigwedge p r_{v}^{-1}(c)$ where $v$ is an arbitrary valuation in $U$ and $c$ is a join-irreducible element of $\mathbf{A} \lg (v)$. We present a concrete example illustrating this using the variety $\mathcal{V}$ generated by the 5 -element non-modular lattice N.

Let the elements of the lattice $\mathbf{N}$ be $0,1,2,3,4$ with $4 \vee 2=4 \vee 3=1$ and $4 \wedge 2=4 \wedge 3=0$. This lattice is generated by the set $\{2,3,4\}$ and any generating set must contain this set of elements. If $\mathcal{V}$ is the lattice variety generated by $\mathbf{N}$, then the only subdirectly irreducible lattices in $\mathcal{V}$ are $\mathbf{N}$ and the 2-element chain $\mathbf{C}_{2}$ with elements $0<1$. So the variety $\mathcal{V}$ covers the variety of distributive lattices in the lattice of all lattice varieties. Now $\mathbf{C}_{2}$ is a homomorphic image of $\mathbf{N}$, so if $p$ and $q$ are lattice terms in the variables $X$ and if $v$ is a valuation to $\mathbf{C}_{2}$ for which $v(p) \neq v(q)$, then there is a $w \in \mathbf{N}^{X}$ for which $w(p) \neq w(q)$. So there is a set $U \subseteq \mathbf{N}^{X}$ for which $\mathbf{F}_{\mathcal{V}}(X)$ is lattice isomorphic to $\mathbf{G e}(X, U)$.

In order to apply Wille's result we need a description of $\bigwedge p r^{-1}(c)$ for $c$ a join-irreducible element of $N$. The following is proved in [6]:

Let $h: \mathbf{F}_{\mathcal{V}}(X) \rightarrow \mathbf{N}$ be an onto homomorphism. For $i \in N$ define $\bar{i}=\bigwedge\{x \in X: h(x) \geq i\}$. Then $h$ is a bounded homomorphism with a lower bound given by $h(\bar{p}) \geq i$ if and only if $\bar{p} \geq \bar{i} \wedge(\overline{2} \vee \overline{4})$ in $\mathbf{F}_{\mathcal{V}}(X)$.

We view $\mathbf{F}_{\mathcal{V}}(X)$ as $\mathbf{G e}(X, U)$ and we let $v$ be any valuation in $U$. Then the projection $p r_{v}$ is the homomorphism of $\mathbf{F}_{\mathcal{V}}(X)$ onto $\mathbf{N}$ that extends $v$. For a nonsingleton congruence class $\bar{p} / \gamma$ we have that a typical join-irreducible element will be of the form $p_{*} \vee(\bar{i} \wedge(\overline{2} \vee \overline{4}))$ where $i$ is join-irreducible in $\mathbf{N}$. If $p$ is a lattice term for which $g\left(p_{*}\right)=g\left(p^{*}\right)$, then the class $\bar{p} / \gamma$ is a singleton since $\mathcal{D} \models p_{*} \approx p^{*}$. If $v \in \mathbf{N}^{X}$ is such that $v\left(p_{*}\right) \neq v\left(p^{*}\right)$, then $\left\{v\left(p_{*}\right), v\left(p^{*}\right)\right\}=$ $\{2,3\}$ since $\mathbf{N} / \theta$ is a distributive lattice for $\theta$ the congruence relation generated by identifying 2 and 3. So for every $\bar{q} \in \bar{p} / \gamma$ we have $v(q) \in\{2,3\}$ since $v\left(p_{*}\right) \leq v(q) \leq v\left(p^{*}\right)$. Hence the entire congruence class $\bar{p} / \gamma$ is embedded in a product of copies of the 2 -element chain $2<3$. This implies that $\bar{p} / \gamma$ is a distributive lattice. Any finite distributive lattice is uniquely determined by its ordered set of join-irreducible elements.

We consider the simplest case in which $|X|=3$ and $g: \mathbf{F}_{\mathcal{V}}(3) \rightarrow \mathbf{F}_{\mathcal{D}}(3)$. The cardinality of $\mathbf{F}_{\mathcal{D}}(3)$ is 18. So $\gamma=\operatorname{ker} g$ has 18 congruence classes. An examination of the 18 elements shows that 11 have the property that $p_{*}=p^{*}$ and so their $\gamma$ classes are singletons. The remaining seven classes $\bar{p} / \gamma$ have as $p_{*}$ either the lower median term $\left(x_{1} \wedge x_{2}\right) \vee\left(x_{1} \wedge x_{3}\right) \vee\left(x_{2} \wedge x_{3}\right)$ or the six duals or permutations of $x_{1} \vee\left(x_{2} \wedge x_{3}\right)$. There are six valuations of $\left\{x_{1}, x_{2}, x_{3}\right\}$ into $\mathbf{N}$, so $\mathbf{F}_{\mathcal{V}}(X)$ is in $\mathbf{N}^{6}$ and each class $\bar{p} / \gamma$ is a sublattice of the distributive lattice $\mathbf{C}_{2}^{6}$. The joinirreducible elements in the $\gamma$ class of the lower median term can be shown to form a six-element antichain. So this class is lattice isomorphic to $\left(\mathbf{C}_{2}\right)^{6}$. Each of the other six congruence classes has a two-element antichain for the ordered set of join-irreducible elements. So each of these congruence classes is isomorphic to $\mathbf{C}_{2} \times \mathbf{C}_{2}$. So all the 18 congruence classes are Boolean lattices of cardinality 1, 4 or 64 . Thus, the cardinality of $\mathbf{F}_{\mathcal{V}}(3)$ is $11+64+6 * 4=99$. The classes interact with one another as in the 18-element free distributive lattice. This analysis provides a reasonably good description of the the structure of $\mathbf{F}_{\mathcal{V}}(3)$.

If we next consider $\mathbf{F}_{\mathcal{V}}(4)$, then the basic analysis is the same. The distributive lattice $\mathbf{F}_{\mathcal{D}}(4)$ has 166 elements. Of the 166 congruence classes of $\gamma$ there are 26 for which $p_{*}=p^{*}$. 
The 140 classes that are not singletons can be grouped by duality and permutation of variables into 12 families. For each of these 12 the ordered set of join-irreducibles can be determined. These ordered sets range in size from 6 to 36 elements. Unlike the case for $\mathbf{F}_{\mathcal{V}}(3)$, these ordered sets are not particularly well-behaved. Even though the distributive lattices that have these ordered sets as their set of join-irreducible elements can be found, the structure of each class is not very transparent. So although the cardinality of $\mathbf{F}_{\mathcal{V}}(4)$ can be determined by this method, it is $540,792,672$, the structure of this lattice can only be described in fairly general terms because of the lack of regularity in the structure of the congruence classes of $\gamma$.

If we consider the variety $\mathcal{W}$ generated by the 5 -element modular nondistributive lattice, then a similar analysis leads to similar results. The lattice $\mathbf{F}_{\mathcal{W}}(3)$ has 28 elements and each of the nonsingleton classes of $\gamma$ well-behaved. But for $\mathbf{F}_{\mathcal{W}}(4)$, although the method of mapping down to $\mathbf{F}_{\mathcal{D}}$ (4) allows us to find the cardinality, which is 19,982, the structure of each class of $\gamma$ is far from transparent.

One difficulty with the method used in Example 2.1 and with the more general technique of partitioning $\mathbf{F}_{\mathcal{V}}(X)$ into congruence classes determined by the canonical map of $\mathbf{F}_{\mathcal{V}}(X)$ onto $\mathbf{F}_{\mathcal{W}}(X)$ for a subvariety $\mathcal{W}$ of $\mathcal{V}$ is that the congruence classes are not 'free' in any obvious sense. We conclude this section with a discussion of a method in which $\mathbf{F}_{\mathcal{V}}(X)$ is decomposed into congruence classes and each class is a free object in a variety intimately related to $\mathcal{V}$.

Let $\mathcal{W}$ be an arbitrary variety in which there are no constant symbols. As in Example 2.1 we consider an equivalence relation $\stackrel{v}{\sim}$ defined on a free algebra $\mathbf{F}_{\mathcal{W}}(X)$ by $\bar{p} \stackrel{v}{\sim} \bar{q}$ if and only if $\operatorname{var}(p)=\operatorname{var}(q)$. When is $\stackrel{v}{\sim}$ a congruence relation? A sufficient condition is that if $\mathcal{W} \models s \approx t$, then $\operatorname{var}(s)=\operatorname{var}(t)$. Identities of this form are called regular and a variety that is presented by regular identities is called a regular variety. If $\stackrel{v}{\sim}$ is a congruence relation of $\mathbf{F}_{\mathcal{W}}(X)$, then the quotient algebra $\mathbf{S}=\mathbf{F}_{\mathcal{W}}(X) / \stackrel{v}{\sim}$ can be represented as an algebra whose universe is the set of nonvoid subsets of $X$ and if $f$ is an $k$-ary operation symbol, then $f^{\mathbf{S}}\left(S_{1}, \ldots, S_{k}\right)=S_{1} \cup \cdots \cup S_{k}$ for $S_{1}, \ldots, S_{k} \subseteq X$. So $\mathbf{S}$ is term equivalent to a join semilattice. We let $\mathcal{S}$ denote the variety of join semilattices but presented with the similarity type of $\mathcal{W}$. Then the canonical homomorphism $g: \mathbf{F}_{\mathcal{W}}(X) \rightarrow \mathbf{F}_{\mathcal{S}}(X)$ determined by $g\left(\bar{x}_{i}\right)=\bar{x}_{i}$ is such that the congruence relation ker $g$ is $\stackrel{v}{\sim}$. Note that each congruence class is in fact a subuniverse of $\mathbf{F}_{\mathcal{W}}(X)$. The classes of ker $g$ interact with each other according to the join semilattice structure on $\mathbf{F}_{\mathcal{S}}(X)$. This interaction is very well-behaved and transparent since it can be viewed as the operation of union on the set of nonvoid subsets of $X$. We describe a general situation in which the individual classes of ker $g$ will have well-behaved internal structure as well. In this situation the structure of $\mathbf{F}_{\mathcal{V}}(X)$ admits a reasonable description.

If $\mathcal{V}$ is an arbitrary variety, then we can form the regularization of $\mathcal{V}$, denoted $R(\mathcal{V})$, which is the variety axiomatized by all the regular identities of $\mathcal{V}$. The variety $\mathcal{V}$ is a subvariety of $R(\mathcal{V})$. We investigate the structure of the free algebras of $R(\mathcal{V})$ by considering the canonical $\operatorname{map}$ of $\mathbf{F}_{R(\mathcal{V})}(X)$ onto $\mathbf{F}_{\mathcal{V}}(X)$.

Let $\mathcal{V}$ be a finitely generated variety that is not regular and that has no constant symbols in its similarity type. Since $\mathcal{V}$ is not regular, there is an $n \geq 2$ and there are terms $s$ and $t$ with $\operatorname{var}(s)=\left\{x_{1}, \ldots, x_{n}\right\}$ and $\operatorname{var}(t)=\left\{x_{1}, \ldots, x_{i}\right\}$ for $i<n$ such that $\mathcal{V} \models s \approx t$. Identifying $x_{1}, \ldots, x_{i}$ with $x$ and identifying $x_{i+1}, \ldots, x_{n}$ with $y$, we have $\mathcal{V} \models s(x, \ldots, x, y \ldots, y) \approx$ $t(x, \ldots, x)$. If we restrict our investigation to the case that $\mathcal{V} \models t(x, \ldots, x) \approx x$, then this situation we then have a binary term $p(x, y)=s(x, \ldots, x, y, \ldots, y)$ for which $\mathcal{V} \models p(x, y) \approx x$ 
witnesses that $\mathcal{V}$ is not a regular variety.

The following theorem of J. Płonka [19] gives the structural decomposition of free algebras in a variety that is the regularization of an irregular variety.

Let $\mathcal{V}$ be a variety with no constant symbols that satisfies an irregular identity of the form $p(x, y) \approx x$. Let $\mathcal{W}=R(\mathcal{V})$ be the regularization of $\mathcal{V}$. Then for $\bar{p} \in \mathbf{F}_{\mathcal{W}}(X)$ the congruence class of $\bar{p} / \stackrel{v}{\sim}$ is a subalgebra of $\mathbf{F}_{\mathcal{W}}(X)$ that is isomorphic to $\mathbf{F}_{\mathcal{V}}(\operatorname{var}(p))$.

An immediate corollary of this theorem is that if $\mathcal{V}$ is also locally finite then

$$
\left|\mathbf{F}_{R(\mathcal{V})}(n)\right|=\sum_{i=1}^{n}\left(\begin{array}{l}
n \\
i
\end{array}\right)\left|\mathbf{F}_{\mathcal{V}}(i)\right| .
$$

We present a constructive proof of Płonka's theorem in the case that the variety $\mathcal{V}$ is finitely generated. We do this by representing $\mathbf{F}_{R(\mathcal{V}}(X)$ as $\mathbf{G e}(X, U)$ for a suitable set $U$ of valuations.

If $\mathbf{A}$ is an algebra and $0 \notin A$, then $\mathbf{A}^{*}$ denotes the algebra having the same similarity type as $\mathbf{A}$, with universe $A \cup\{0\}$, and operations given by

$$
f^{\mathbf{A}^{*}}\left(a_{1}, \ldots, a_{k}\right)= \begin{cases}0 & \text { if } 0 \in\left\{a_{1}, \ldots, a_{k}\right\} \\ f^{\mathbf{A}}\left(a_{1}, \ldots, a_{k}\right) & \text { otherwise. }\end{cases}
$$

The element 0 is called an absorbing element of $\mathbf{A}^{*}$.

Let $\mathbf{A}$ be a finite algebra with no constant symbols in its similarity type and suppose $\mathbf{A} \models p(x, y) \approx x$ for a term $p$ with $\operatorname{var}(p)=\{x, y\}$. If $\mathcal{V}$ is the variety generated by $\mathbf{A}$, then $\mathcal{V}$ satisfies the hypotheses of Płonka's theorem. By results of H. Lakser, R. Padmanabhan, and C. Platt [17], the variety $R(\mathcal{V})$ is generated by the algebra $\mathbf{A}^{*}$. Therefore, the algebra $\mathbf{F}_{R(\mathcal{V})}(X)$ is isomorphic to $\mathbf{G e}(X, U)$ where $U$ consists of all $v: X \rightarrow \mathbf{A}^{*}$. Let $Z$ be any nonvoid subset of $X$. Without loss of generality we let $Z=\left\{x_{1}, \ldots, x_{m}\right\}$. Form

$$
U_{Z}=\left\{v \in U: v\left(x_{i}\right) \in A \text { for all } x_{i} \in Z\right\} .
$$

For $1 \leq i \leq m$ let $y_{i}$ denote the term $p\left(x_{i}, p\left(x_{1}, p\left(x_{2}, p\left(\ldots, p\left(x_{m-1}, x_{m}\right) \ldots\right)\right)\right)\right)$. Then $\mathcal{V} \models$ $x_{i} \approx y_{i}$ and $\operatorname{var}\left(y_{i}\right)=Z$. For $v \in U_{Z}$ and $x_{i} \in Z$ we have $v\left(x_{i}\right)=v\left(y_{i}\right)$. If $w \in U-U_{Z}$, then there is an $x_{j} \in Z$ for which $w\left(x_{j}\right)=0$. Hence $w\left(y_{i}\right)=0$ for all $1 \leq i \leq m$. Let $t$ be any term with $\operatorname{var}(t)=Z$. We write $t$ as $t\left(x_{1}, \ldots, x_{m}\right)$. For all $v \in U_{Z}$ we have $v(t)=v\left(t\left(x_{1}, \ldots, x_{m}\right)\right)=v\left(t\left(y_{1}, \ldots, y_{m}\right)\right) \in A$. For all $w \in U-U_{Z}$ we have $w(t)=0$. From these observations it follows that the congruence class and subuniverse $\bar{t} / \stackrel{v}{\sim}$ is generated by $\bar{y}_{1}, \ldots, \bar{y}_{m}$. Moreover, $\bar{t} / \stackrel{v}{\sim}$ can be embedded in $\mathbf{G e}\left(X, U_{Z}\right)$ since $w(s)=w(t)$ for every $w \in U-U_{Z}$ and $s$ having $\operatorname{var}(s)=Z$. Now $\bar{y}_{i}$ and $\bar{x}_{i}$ agree on all $v \in U_{Z}$. So the subalgebra of $\mathbf{G e}\left(X, U_{Z}\right)$ generated by $\bar{y}_{1}, \ldots, \bar{y}_{m}$ is isomorphic to the subalgebra of $\mathbf{G e}\left(X, U_{Z}\right)$ generated by $Z=\left\{x_{1}, \ldots, x_{m}\right\}$. This latter algebra is isomorphic to $\operatorname{Ge}\left(Z, A^{Z}\right)$. Since $\operatorname{Ge}\left(Z, A^{Z}\right) \cong$ $\mathbf{F}_{\mathcal{V}}(Z)$ we conclude that $\bar{t} / \stackrel{v}{\sim}$ is isomorphic to $\mathbf{F}_{\mathcal{V}}(Z)$.

For example, let $\mathcal{B}$ be the variety of Boolean algebras considered in Example 1.9. The variety $\mathcal{B}$ is generated by the 2 -element Boolean algebra $\mathbf{B}_{2}$. We can eliminate the constant symbols 0 and 1 by using the unary terms $x_{1} \wedge x_{1}^{\prime}$ and $x_{1} \vee x_{1}^{\prime}$ in their stead. If $p(x, y)$ denotes the term $(x \vee y) \wedge x$, then $\mathcal{B} \models p(x, y) \approx x$. If $\mathcal{W}$ denotes the regularization $R(\mathcal{B})$ of Boolean algebras, then as seen in the previous paragraph, $\mathcal{W}$ is generated by the 3 -element algebra 
obtained by adjoining an absorbing element to $\mathbf{B}_{2}$. Each congruence class $\bar{t} / \stackrel{v}{\sim}$ in $\mathbf{F}_{\mathcal{W}}(X)$ is isomorphic to the free Boolean algebra $\mathbf{F}_{\mathcal{B}}(\operatorname{var}(t))$. For $\bar{s}, \bar{t} \in \mathbf{F}_{\mathcal{W}}(X)$ and term $q(x, y)$, we have $q(\bar{s}, \bar{t}) / \stackrel{v}{\sim}=\bar{r} / \stackrel{v}{\sim}$ where $r$ is any term with $\operatorname{var}(r)=\operatorname{var}(s) \cup \operatorname{var}(t)$. As observed in Example $1.9\left|\mathbf{F}_{\mathcal{B}}(m)\right|=2^{2^{m}}$. Thus,

$$
\mathbf{F}_{R(\mathcal{B})}(n)=\sum_{i=1}^{n}\left(\begin{array}{l}
n \\
i
\end{array}\right) 2^{2^{i}} .
$$

\section{Structure via inclusion-exclusion}

In the previous section we investigated the structure of a free algebra by decomposing it into the disjoint blocks of a congruence relation that is the kernel of a homomorphism onto a free algebra in a particular subvariety. In this section we decompose a free algebra into overlapping canonically defined subalgebras. The homogeneity of these subalgebras allows for the inclusion-exclusion principle to be used to express the cardinality of the free algebra in terms of the cardinalities of these subalgebras. We present some examples of varieties in which the subalgebras have some interesting structure of their own and for which we can either determine their cardinality or else express the cardinality in terms of the cardinalities of some sets of some familiar combinatorial structures.

Throughout this section we let $X=\left\{x_{1}, \ldots, x_{n}\right\}$ be a finite set of variables. For a language (or similarity type) $\mathcal{L}$ let $\mathrm{T}_{\mathcal{L}}(X)$ denote the set of all terms that can be built from $X$ using operation symbols from $\mathcal{L}$. We often write $\mathrm{T}(n)$ for $\mathrm{T}_{\mathcal{L}}(X)$. In this section $\mathcal{L}$ will always be a language in which there are no constant symbols. Since a nullary constant operation can always be represented by a constant unary operation, this restriction on $\mathcal{L}$ does not result in any loss of generality.

3.1 Definition For $t \in \mathrm{T}_{\mathcal{L}}(X)$ we define the right-most variable $\mathrm{rv}(t)$ of $t$ inductively as follows:

$$
\operatorname{rv}(t)= \begin{cases}x_{i} & \text { if } t=x_{i} \in X \\ \operatorname{rv}\left(t_{m}\right) & \text { if } t=f\left(t_{1}, \ldots, t_{m}\right) \text { for } f \in \mathcal{L} \text { and } t_{1}, \ldots, t_{m} \in \mathrm{T}_{\mathcal{L}}(X) .\end{cases}
$$

For $1 \leq i \leq n$, let $\mathrm{T}_{i}(n)=\left\{t \in \mathrm{T}(n): \operatorname{rv}(t)=x_{i}\right\}$ and let $\overline{\mathrm{T}}_{i}(n)$ be the set of elements $\left\{\bar{t} \in \mathbf{F}_{\mathcal{V}}(n): t \in \mathrm{T}_{i}(n)\right\}$. It is easily seen that each $\overline{\mathrm{T}}_{i}(n)$ is a subuniverse of $\mathbf{F}_{\mathcal{V}}(X)$; we write $\overline{\mathbf{T}}_{i}(n)$ for the corresponding subalgebra of $\mathbf{F}_{\mathcal{V}}(n)$.

Note that the algebras $\overline{\mathbf{T}}_{i}(n)$ need not be disjoint. We use the $\overline{\mathbf{T}}_{i}(n)$ in our decomposition of $\mathbf{F}_{\mathcal{V}}(X)$. The intersections of the $\overline{\mathrm{T}}_{i}(n)$ will also play a role. We write $\overline{\mathrm{T}}_{\leq \ell}(n)$ for $\bigcap_{1 \leq i \leq \ell} \overline{\mathrm{T}}_{i}(n)$. Each $\overline{\mathrm{T}}_{\leq \ell}(n)$ is a subuniverse and $\overline{\mathbf{T}}_{\leq \ell}(n)$ denotes the corresponding subalgebra.

All the $\overline{\mathbf{T}}_{i}(n)$ are isomorphic to each other. Every permutation of $X$ extends to an automorphism of $\mathbf{F}_{\mathcal{V}}(X)$. From this it follows that if $1 \leq i_{1}<\cdots<i_{\ell} \leq n$, then $\overline{\mathbf{T}}_{i_{1}}(n) \cap$ $\overline{\mathbf{T}}_{i_{2}}(n) \cap \cdots \cap \overline{\mathbf{T}}_{i_{\ell}}(n) \cong \overline{\mathbf{T}}_{\leq \ell}(n)$.

3.2 Theorem Let $\mathcal{V}$ be a locally finite variety in a similarity type that has no constant symbols. For every positive integer $n$ we have

$$
\left|\mathbf{F}_{\mathcal{V}}(n)\right|=\sum_{\ell=1}^{n}(-1)^{\ell-1}\left(\begin{array}{l}
n \\
\ell
\end{array}\right)\left|\overline{\mathrm{T}}_{\leq \ell}(n)\right| .
$$


Proof First note that $\mathcal{V}$ is locally finite, and hence $\mathrm{F}_{\mathcal{V}}(n)$ is a finite set. Every element of $\mathbf{F}_{\mathcal{V}}(n)$ is of the form $\bar{t}$ for some $t \in \mathrm{T}(n)$. There are no constant symbols in the similarity type of $\mathcal{V}$ and so $t$ has a right-most variable $\operatorname{rv}(t)$, that is, $t \in \mathrm{T}_{i}(n)$ for some $i$. Thus,

$$
\mathrm{F}_{\mathcal{V}}(n)=\bigcup_{1 \leq i \leq n} \overline{\mathrm{T}}_{i}(n)
$$

Applying the inclusion-exclusion principle, we have

$$
\left|\mathbf{F}_{\mathcal{V}}(n)\right|=\sum\left\{(-1)^{\ell-1}\left|\overline{\mathrm{T}}_{i_{1}}(n) \cap \overline{\mathrm{T}}_{i_{2}}(n) \cap \cdots \cap \overline{\mathrm{T}}_{i_{\ell}}(n)\right|: 1 \leq i_{1}<\cdots<i_{\ell} \leq n\right\} .
$$

As we have observed, $\overline{\mathbf{T}}_{i_{1}}(n) \cap \overline{\mathbf{T}}_{i_{2}}(n) \cap \cdots \cap \overline{\mathbf{T}}_{i_{\ell}}(n)$ is isomorphic to $\overline{\mathbf{T}}_{\leq \ell}(n)$ so

$$
\left|\overline{\mathrm{T}}_{i_{1}}(n) \cap \overline{\mathrm{T}}_{i_{2}}(n) \cap \cdots \cap \overline{\mathrm{T}}_{i_{\ell}}(n)\right|=\left|\overline{\mathrm{T}}_{\leq \ell}(n)\right| .
$$

For each $1 \leq \ell \leq n$ there are $\left(\begin{array}{l}n \\ \ell\end{array}\right)$ sets of the form $\overline{\mathrm{T}}_{i_{1}}(n) \cap \overline{\mathrm{T}}_{i_{2}}(n) \cap \cdots \cap \overline{\mathrm{T}}_{i_{\ell}}(n)$, each of size $\left|\overline{\mathrm{T}}_{\leq \ell}(n)\right|$, thereby yielding the desired formula for $\left|\mathbf{F}_{\mathcal{V}}(n)\right|$.

Although the formal appearance of Theorem 3.2 is appealing, it is not immediately clear if the result has any content. For example, in any variety of groups or lattices, $\overline{\mathrm{T}}_{i}(n)=\mathrm{F}_{\mathcal{V}}(n)$ for every $1 \leq i \leq n$. For such varieties the theorem tells us nothing. For other varieties, although the $\overline{\mathrm{T}}_{i}(n)$ are proper subsets of $\mathrm{F}_{\mathcal{V}}(n)$, the structure of the subalgebra $\overline{\mathbf{T}}_{i}(n)$ is difficult to determine. Nonetheless, there are varieties in which the algebras $\overline{\mathbf{T}}_{i}(n)$ have an interesting describable structure and for which Theorem 3.2 can be used to provide significant information about the cardinalities of finitely generated free algebras. In what follows we present two varieties in which this is the case. Each is based on an algebraic coding of ordered sets as presented in [3] and [4].

3.3 Definition Let $P=\langle P, \leq, 1\rangle$ be a ordered set with a top element 1 . We form the algebra $\mathbf{H}(P)=\langle P, \cdot\rangle$ with universe $P$ and binary operation $\cdot$ given by

$$
x \cdot y= \begin{cases}1 & \text { if } x \leq y \\ y & \text { otherwise }\end{cases}
$$

and the algebra $\mathbf{J}(P)=\langle P, \cdot\rangle$ with universe $P$ and binary operation $\cdot$ given by

$$
x \cdot y= \begin{cases}y & \text { if } x \leq y \\ 1 & \text { otherwise }\end{cases}
$$

By $\mathcal{H}$ and $\mathcal{J}$ we denote the varieties generated by all $\mathbf{H}(P)$ and all $\mathbf{J}(P)$, where $P$ ranges over all ordered sets with a top element.

The variety $\mathcal{H}$ is a subvariety of the variety of all Hilbert algebras, which is the variety generated by all $\{\rightarrow, 1\}$-subreducts of the varieties of Brouwerian semilattices. Hilbert algebras have received considerable attention in the algebraic logic literature. A standard reference on the basic properties of Hilbert algebras is A. Diego's monograph [9]. A Hilbert algebra includes the constant 1 in the similarity type. However, since Hilbert algebras satisfy the identity $x \rightarrow x=1$, this constant can be omitted from the similarity type. For $\mathcal{H}$ we write for the binary operation symbol $\rightarrow$ and omit the constant 1 from the similarity type. 
Members of $\mathcal{J}$ are called join algebras. The only work on join algebras that I am aware of is [4].

It is not hard to argue that every algebra in $\mathcal{H}$ or in $\mathcal{J}$ has an equationally definable order relation. For elements in an algebra in $\mathcal{H}$ we have $x \leq y$ if and only if $x y=x x$ while for algebras in $\mathcal{J}$ the order is given by $x \leq y$ if and only if $x y=y$.

3.4 Definition An algebra $\mathbf{A}$ in $\mathcal{H}$ is called pure if it is of the form $\mathbf{H}(P)$ for some ordered set $P$ having a top element. A pure algebra in $\mathcal{J}$ is defined analogously.

Both $\mathcal{H}$ and $\mathcal{J}$ are generated by their pure members. Both $\mathcal{H}$ and $\mathcal{J}$ are known to be locally finite varieties. The subdirectly irreducible algebras in each variety have been characterized: they are the pure algebras with underlying ordered set $P$ of the form $Q \oplus 1$, where $Q$ is an arbitrary ordered set.

There are some differences between $\mathcal{H}$ and $\mathcal{J}$. Notably, $\mathcal{H}$ is a congruence distributive variety and its type set, in the sense of tame congruence theory, is $\{\mathbf{3}\}$ while $\mathcal{J}$ satisfies no nontrivial congruence identities and its type set is $\{\mathbf{5}\}$.

Another important difference between $\mathcal{H}$ and $\mathcal{J}$ is that the variety $\mathcal{J}$ of join algebras is generated by $\mathbf{J}(C)$, where $C$ is the 3 -element chain. The only proper nontrivial subvariety of $\mathcal{J}$ is the variety of semilattices. These results are proved in [4]. The variety $\mathcal{H}$ on the other hand, is not finitely generated and has $2^{\aleph_{0}}$ subvarieties. The least nontrivial subvariety of $\mathcal{H}$ is the variety $\mathcal{I}$ of implication algebras discussed in Example 1.10. These results and others for $\mathcal{H}$ are also given in [4].

We investigate the structure of free algebras $\mathbf{F}_{\mathcal{H}}(n)$ and $\mathbf{F}_{\mathcal{J}}(n)$ by describing the subalgebras $\overline{\mathbf{T}}_{i}(n)$.

3.5 Definition Let $t \in \mathrm{T}_{\mathcal{L}}(n)$ be an arbitrary term for $\mathcal{L}=\{\cdot\}$.

The binary relation $\operatorname{re}(t)$ on $\operatorname{var}(t)$ is defined inductively by:

$$
\operatorname{re}(t)= \begin{cases}\left\{\left(x_{i}, x_{i}\right)\right\} & \text { if } t=x_{i} \\ \operatorname{re}(p) \cup \operatorname{re}(q) \cup\{(\operatorname{rv}(p), \operatorname{rv}(q))\} & \text { if } t=p \cdot q\end{cases}
$$

The transitive closure of re $(t)$ is denoted qo $(t)$.

A quasi-order on a set $Z$ is any reflexive transitive binary relation on $Z$. If $\sigma$ is a quasiorder on $Z$, then the quotient $Z / \sigma$ is an ordered set in which $a / \sigma \leq b / \sigma$ if and only if $(a, b) \in \sigma$. The ordered set $Z / \sigma$ has a top element if and only if there is a $z \in Z$ such that $(a, z) \in \sigma$ for all $a \in Z$. If $t \in \mathrm{T}_{\mathcal{L}}(n)$, then qo( $\left.t\right)$ is a quasi-order on the set $\operatorname{var}(t)$ in which $\mathrm{rv}(t) / \mathrm{qo}(t)$ is the top element of the ordered set $\operatorname{var}(t) / \mathrm{qo}(t)$.

3.6 Lemma Let $\sigma$ be any quasi-order on a set $Y \subseteq X$ such that $Y / \sigma$ has a top element. Then there exists $t \in \mathrm{T}_{\mathcal{L}}(X)$ such that $\mathrm{qo}(t)=\sigma$.

Proof Let $\left(x_{i_{1}}, x_{j_{1}}\right),\left(x_{i_{2}}, x_{j_{2}}\right),\left(x_{i_{3}}, x_{j_{3}}\right), \ldots,\left(x_{i_{k}}, x_{j_{k}}\right)$ be any list of the elements of $\sigma$ subject only to the constraint that $x_{j_{1}} / \sigma$ is the top element of $Y / \sigma$. Let

$$
t=\left(x_{i_{k}} x_{j_{k}}\right)\left(\ldots\left(x_{i_{3}} x_{j_{3}}\right)\left(\left(x_{i_{2}} x_{j_{2}}\right)\left(x_{i_{1}} x_{j_{1}}\right)\right) \ldots\right) .
$$

Clearly $\operatorname{var}(t)=Y$. It is immediate from the recursive definition of the operator qo that $\mathrm{qo}(t)=\sigma$. 
We now restrict our discussion to the variety $\mathcal{J}$. The next lemma is proved by considering valuations of $X$ into the 3 -element pure join algebra $\mathbf{H}(C)$, which generates $\mathcal{J}$.

3.7 Lemma Let $s, t \in \mathrm{T}_{\mathcal{L}}(n)$.

(1) $\mathcal{J} \models s \approx t$ iff $\mathrm{qo}(s)=\mathrm{qo}(t)$.

(2) $\mathcal{J} \models s t \approx t$ iff $\mathrm{qo}(s) \subseteq \mathrm{qo}(t)$.

We have $\overline{\mathrm{T}}_{i}(n)=\left\{\bar{t} \in \mathbf{F}_{\mathcal{J}}(n): \operatorname{rv}(t)=x_{i}\right\}$ and that $\overline{\mathbf{T}}_{i}(n)$ is the subalgebra of $\mathbf{F}_{\mathcal{J}}(n)$ with universe $\overline{\mathrm{T}}_{i}(n)$. It can be argued that $\mathcal{J}$ is a regular variety (in the sense of the previous section), that is, if $\mathcal{J} \models s \approx t$, then $\operatorname{var}(s)=\operatorname{var}(t)$. In particular, if $\bar{s} \in \overline{\mathrm{T}}_{i}(n)$, then $x_{i} \in \operatorname{var}(s)$.

For $1 \leq i \leq n$ let

$$
\begin{aligned}
& \mathrm{Q}_{i}(n)=\left\{\sigma: \quad \exists Y \subseteq X, x_{i} \in Y, \sigma \text { is a quasi-order on } Y,\right. \\
&\text { and } \left.x_{i} / \sigma \text { is the top element of } Y / \sigma\right\} .
\end{aligned}
$$

For $\sigma_{1}$ and $\sigma_{2} \in \mathrm{Q}_{i}(n)$ with $\sigma_{k}$ defined on $Y_{k} \subseteq X$ for $k=1,2$, let $\sigma_{1} \cdot \sigma_{2}$ denote the smallest quasi-order on $Y_{1} \cup Y_{2}$ that contains both $\sigma_{1}$ and $\sigma_{2}$. Then necessarily $\sigma_{1} \cdot \sigma_{2} \in \mathrm{Q}_{i}(n)$ and $\sigma_{1} \cdot \sigma_{2}$ is the transitive closure of $\sigma_{1} \cup \sigma_{2}$. Let $\mathbf{Q}_{i}(n)$ denote the algebra $\left\langle\mathrm{Q}_{i}(n), \cdot\right\rangle$. We note that the algebra $\mathbf{Q}_{i}$ is, in fact, a semilattice since the operation - on $\mathrm{Q}_{i}$ is idempotent, commutative, and associative.

3.8 Theorem The map $\bar{t} \mapsto \mathrm{qo}(t)$ is an isomorphism from $\overline{\mathbf{T}}_{i}(n)$ onto $\mathbf{Q}_{i}(n)$.

Proof Lemmas 3.6 and 3.7 show the map is well-defined, one-to-one and onto $\mathrm{Q}_{i}(n)$. It remains to show that $\mathrm{qo}(s \cdot t)=\mathrm{qo}(s) \cdot \mathrm{qo}(t)$ for all $\bar{s}$ and $\bar{t}$ in $\overline{\mathrm{T}}_{i}(n)$. Let $\operatorname{rv}(s)=x_{\ell}$ and $\operatorname{rv}(t)=x_{m}$. By the definition of qo we have that qo $(s \cdot t)$ is the transitive closure of $\mathrm{qo}(s) \cup \mathrm{qo}(t) \cup\left\{\left(x_{\ell}, x_{m}\right)\right\}$. We have $\left(x_{\ell}, x_{i}\right) \in \mathrm{qo}(s)$ since $\bar{s} \in \overline{\mathrm{T}}_{i}(n)$. Also $\left(x_{i}, x_{m}\right) \in \mathrm{qo}(t)$ since $t \in \overline{\mathrm{T}}_{i}(n)$ and $\operatorname{rv}(t)=x_{m}$. So $\left(x_{\ell}, x_{m}\right)$ is in the transitive closure of qo $(s) \cup$ qo $(t)$. Hence the transitive closure of $\mathrm{qo}(s) \cup \mathrm{qo}(t) \cup\left\{\left(x_{\ell}, x_{m}\right)\right\}$ is the same as the transitive closure of $\mathrm{qo}(s) \cup \mathrm{qo}(t)$, which is $\mathrm{qo}(s) \cdot \mathrm{qo}(t)$.

From Theorem 3.8 it follows that $\mathbf{Q}_{i}(n)$ is a join algebra. The induced order on $\mathbf{Q}_{i}(n)$ is that of containment. Therefore, $\bar{s} \leq \bar{t}$ in $\overline{\mathbf{T}}_{i}(n)$ if and only if $\mathrm{qo}(s) \subseteq \mathrm{qo}(t)$. Actually, from Lemma 3.7 we see that for arbitrary $\bar{s}, \bar{t} \in \overline{\mathrm{T}}_{i}(n)$ it is the case that $\bar{s} \leq \bar{t}$ if and only if $\mathrm{qo}(s) \subseteq \mathrm{qo}(t)$.

Let $q_{k}$ denote the number of quasi-orders on $\{1,2, \ldots, k\}$. It is known that $q_{k}$ is also equal to the number of topologies on $\{1,2, \ldots, k\}$. For example, the values of $q_{k}$ for $k=1,2,3,4$ are $1,4,29,355$ respectively. We let $q_{0}=1$.

\subsection{Theorem}

$$
\left|\mathrm{F}_{\mathcal{J}}(n)\right|=\sum_{i=0}^{n-1} q_{i}\left(\begin{array}{c}
n \\
i
\end{array}\right)\left(2^{n-i}-1\right) .
$$

Proof We have

$$
\left|\mathbf{F}_{\mathcal{J}}(n)\right|=\sum_{\ell=1}^{n}(-1)^{(\ell-1)}\left(\begin{array}{l}
n \\
\ell
\end{array}\right)\left|\overline{\mathrm{T}}_{\leq \ell}(n)\right|
$$


by Theorem 3.2. The cardinality of $\overline{\mathbf{T}}_{\leq \ell}(n)$ is equal to $\left|\mathrm{Q}_{1}(n) \cap \cdots \cap \mathrm{Q}_{\ell}(n)\right|$ by Theorem 3.8. Let $\sigma$ be a quasi-order on a set $Y \subseteq X$ for which $Y / \sigma$ has a top element. Let $Z \subseteq Y$ be be the set of all $x_{i}$ for which $x_{i} / \sigma$ is the top element in $Y / \sigma$. Then $\sigma=\tau \cup(Y \times Z)$ where $\tau$ is a quasi-order on $Y-Z$. Conversely, if $\emptyset \neq Z \subseteq Y \subseteq X$ and $\tau$ is a quasi-order on $Y-Z$, then $\sigma=\tau \cup(Y \times Z)$ is a quasi-order on $Y$ for which $Y / \sigma$ has a maximal element consisting of $x_{k} / \sigma$ for every $x_{k} \in Z$. For $\sigma \in \mathrm{Q}_{1}(n) \cap \cdots \cap \mathrm{Q}_{\ell}(n)$ there are $\left(\begin{array}{c}n-\ell \\ i\end{array}\right)$ choices for the set $Y-Z$ if $|Y-Z|=i$. For a given $i$, with $0 \leq i \leq n-\ell$, there are $q_{i}$ choices for a quasi-order on $Y-Z$. The set $Z$ must contain $\left\{x_{1}, \ldots, x_{\ell}\right\}$ so there are $2^{n-\ell-i}$ ways to choose the remaining elements of $Z$. Therefore

$$
\left|\mathrm{Q}_{1}(n) \cap \cdots \cap \mathrm{Q}_{\ell}(n)\right|=\sum_{i=0}^{n-\ell} q_{i}\left(\begin{array}{c}
n-\ell \\
i
\end{array}\right) 2^{n-\ell-i} .
$$

This implies

$$
\left|\mathbf{F}_{\mathcal{J}}(n)\right|=\sum_{\ell=1}^{n}(-1)^{(\ell-1)}\left(\begin{array}{c}
n \\
\ell
\end{array}\right)\left(\sum_{i=0}^{n-\ell} q_{i}\left(\begin{array}{c}
n-\ell \\
i
\end{array}\right) 2^{n-\ell-i}\right) .
$$

We interchange the order of summation and use the formula $\left(\begin{array}{c}n \\ \ell\end{array}\right)\left(\begin{array}{c}n-\ell \\ i\end{array}\right)=\left(\begin{array}{c}n \\ i\end{array}\right)\left(\begin{array}{c}n-i \\ n-\ell-i\end{array}\right)$ to obtain

$$
\left|\mathbf{F}_{\mathcal{J}}(n)\right|=\sum_{i=0}^{n-1} q_{i}\left(\begin{array}{c}
n \\
i
\end{array}\right)\left(\sum_{\ell=1}^{n-i}(-1)^{\ell-1}\left(\begin{array}{c}
n-i \\
n-\ell-1
\end{array}\right) 2^{n-\ell-i}\right) .
$$

The inner summation simplifies to $2^{n-i}-1$. Thus,

$$
\left|\mathbf{F}_{\mathcal{J}}(n)\right|=\sum_{i=0}^{n-1} q_{i}\left(\begin{array}{c}
n \\
i
\end{array}\right)\left(2^{n-i}-1\right) .
$$

For example, if we use the previously mentioned values of $q_{i}$ for $1 \leq i \leq 4$, then we see that the values of $\left|\mathrm{F}_{\mathcal{J}}(n)\right|$ for $1 \leq n \leq 5$ are 1, 5, 28, 231 and 3031 respectively.

The formula in Theorem 3.9 involves $q_{n}$, the number of quasi-orders on an $n$-element set. Although no simple formula for the value of $q_{n}$ is known, asymptotic estimates do exist. By means of these asymptotics we can obtain the following bounds on the cardinalities of free join algebras.

3.10 Theorem There exists a positive constant c such that for all sufficiently large n,

$$
2^{\frac{n^{2}}{4}+n-c \lg n} \leq\left|\mathrm{F}_{\mathcal{J}}(n)\right| \leq 2^{\frac{n^{2}}{4}+n+c \lg n}
$$

We next consider the structure of free algebras $\mathbf{F}_{\mathcal{V}}(n)$ for $\mathcal{V}$ an arbitrary subvariety of $\mathcal{H}$. As with the variety $\mathcal{J}$ we provide a detailed description of the subalgebras $\overline{\mathbf{T}}_{i}(n)$. From this description we show that $\overline{\mathbf{T}}_{\leq \ell}(n)$ is a direct power of the 2-element implication algebra $\mathbf{C}_{2}$, and we determine the exponent in this direct power. So with Theorem 3.2 we can, in principle, find an expression for the cardinality of $\mathbf{F}_{\mathcal{V}}(n)$ when $\mathcal{V}$ is a subvariety of $\mathcal{H}$.

Recall that a subdirectly irreducible algebra $\mathbf{A} \in \mathcal{H}$ has an element $e \prec 1$ with $a \leq e$ for all $a \in A, a \neq 1$. The element $e$ is irreducible in the sense that if $t^{\mathbf{A}}\left(a_{1}, \ldots, a_{n}\right)=e$, then 
$e$ appears among the $a_{i}$. Hence if $v$ is a valuation mapping to the subdirectly irreducible algebra $\mathbf{A}$, then $e \in v(X)$. We also note that the set $\{1, e\}$ is a subuniverse of $\mathbf{A}$ and the corresponding subalgebra is isomorphic to the 2-element implication algebra $\mathbf{C}_{2}$. The algebra $\mathbf{C}_{2}$ is a pure algebra in $\mathcal{H}$ since it is $\mathbf{H}(P)$ for $P$ the 2-element chain.

The following facts about valuations into pure algebras in $\mathcal{H}$ are easily established using nothing more than definitions.

3.11 Lemma Let $v$ be a valuation into a pure algebra $\mathbf{A}$ in $\mathcal{H}$ and let $t$ be a term in $\mathrm{T}_{\mathcal{L}}(n)$ with $\operatorname{rv}(t)=x_{i}$.

(1) $v(t) \in\{1, v(\operatorname{rv}(t))\}$.

(2) If $\bar{t} \in \overline{\mathrm{T}}_{i}(n) \cap \overline{\mathrm{T}}_{j}(n)$ and $v(t) \neq 1$, then $v\left(x_{i}\right)=v\left(x_{j}\right)$.

(3) $\mathcal{H} \models \operatorname{rv}(t) \leq t$.

Let $\mathcal{V}$ be a subvariety of $\mathcal{H}$ and $\mathcal{V}_{\mathrm{SI}}$ the class of finitely generated subdirectly irreducible algebras in $\mathcal{V}$. Since $\mathcal{V}$ is locally finite, every algebra in $\mathcal{V}_{\text {SI }}$ is finite. By Corollary 1.4 the set $\operatorname{val}\left(X, \mathcal{V}_{\mathrm{SI}}\right)$ is free for $\mathcal{V}$. Hence $E\left(\operatorname{val}\left(X, \mathcal{V}_{\mathrm{SI}}\right)\right)$ is also free for $\mathcal{V}$ by Lemma 1.7.

Now, from Lemma 3.11, if $v$ is a valuation in $E=E\left(\operatorname{val}\left(X, \mathcal{V}_{\mathrm{SI}}\right)\right)$ and $t \in \mathrm{T}_{i}(n)$, then $v(t) \in\left\{1, v\left(x_{i}\right)\right\}$. If $\bar{s}, \bar{t} \in \overline{\mathrm{T}}_{i}(n)$ with $\bar{s} \neq \bar{t}$, then there exists $v \in E$ with $v\left(x_{i}\right) \leq e$ and $\{v(s), v(t)\}=\left\{1, v\left(x_{i}\right)\right\}$. By composing $v$ with an endomorphism of $\mathbf{A} \lg (v)$ that maps $v\left(x_{i}\right)$ to $e$ we can find a $w \in E$ for which $\{v(s), v(t)\}=\{1, e\}$.

3.12 Definition Let $\mathcal{V}$ be a subvariety of $\mathcal{H}$. We adopt the following notation:

$$
E(\mathcal{V}):=E\left(\operatorname{val}\left(X, \mathcal{V}_{\mathrm{SI}}\right)\right)
$$

For $1 \leq \ell \leq n$,

$$
E(\mathcal{V})_{\ell}:=\left\{v \in E\left(\operatorname{val}\left(X, \mathcal{V}_{\mathrm{SI}}\right)\right): v\left(x_{\ell}\right)=e\right\}
$$

and

$$
E(\mathcal{V})_{\leq \ell}:=\left\{v \in E\left(\operatorname{val}\left(X, \mathcal{V}_{\mathrm{SI}}\right)\right): v\left(x_{i}\right)=e \text { for } 1 \leq i \leq \ell\right\}
$$

When the variety $\mathcal{V}$ is clear from the context or is not important we write $E, E_{\ell}$ and $E_{\leq \ell}$.

Note that $E_{\leq \ell}=E_{1} \cap \cdots \cap E_{\ell}$ follows from the definition and that $E=E_{1} \cup \cdots \cup E_{n}$ follows from the observation that $e \in v(X)$ for every $v \in E$.

For any subvariety $\mathcal{V}$ of $\mathcal{H}$. we know $\mathbf{F}_{\mathcal{V}}(X)$ is isomorphic to $\mathbf{G e}(X, E(\mathcal{V}))$. Since the valuations in $E_{i}$ serve to separate the elements of $\overline{\mathbf{T}}_{i}(n)$, we have $\overline{\mathbf{T}}_{i}(n)$ embedded in $\mathbf{G e}\left(X, E_{i}\right)$. From Lemma 3.11(3) it follows that if $v \in E_{i}$ and $t \in \mathrm{T}_{i}(n)$, then $v(t) \geq v\left(x_{i}\right) \geq e$. This means that the embedding of $\overline{\mathbf{T}}_{i}(n)$ into $\mathbf{G e}\left(X, E_{i}\right)$ is actually an embedding into $\mathbf{C}_{2}^{E_{i}}$. It can be argued that this embedding is onto $\mathbf{C}_{2}^{E_{i}}$. One way this can be done is to construct for every $v \in E_{i}$ a term $t_{v}$ such that $v\left(t_{v}\right)=1$ and $w\left(t_{v}\right)=e$ for every $w \in E_{i}$ with $w \neq v$. The construction makes use of the ordered set of elements $v(X)$ in the subdirectly irreducible algebra $\operatorname{Alg}(v)$ and is similar in spirit to that given in Lemma 3.6. That the valuations in $E_{i}$ are pairwise nonequivalent is also used in this argument. Then by means of the term $(x \cdot y) \cdot y$, which behaves as semilattice join on the ordered set $e<1$, it is possible to form for every element $c$ of $\mathbf{C}_{2}^{E_{i}}$ a term $t \in \mathrm{T}_{i}$ built from the appropriate $t_{v}$ for which $w(t)=c(w)$ for all $w \in E_{i}$. Thus we have the following. 
3.13 Lemma For any subvariety $\mathcal{V}$ of $\mathcal{H}$ the algebra $\overline{\mathbf{T}}_{\leq \ell}(n)$ is isomorphic to $\mathbf{C}_{2}^{\left|E_{\leq \ell}\right|}$ where $\mathbf{C}_{2}=\mathbf{H}(P)$ for $P$ the 2-element chain $e<1$.

A stronger formulation of this lemma is given in [3] where the variety $\mathcal{H}$ is replaced by the variety of all Hilbert algebras.

Theorem 3.2 and Lemma 3.13 provide the following expression for the cardinality of a finitely generated free algebra $\mathbf{F}_{\mathcal{V}}(n)$ in a subvariety $\mathcal{V}$ of $\mathcal{H}$.

3.14 Theorem For every variety $\mathcal{V} \subseteq \mathcal{H}$ and every positive integer $n$

$$
\left|\mathrm{F}_{\mathcal{V}}(n)\right|=\sum_{\ell=1}^{n}(-1)^{\ell-1}\left(\begin{array}{l}
n \\
\ell
\end{array}\right) 2^{\mid E_{\leq \ell \mid}}
$$

So, for a variety $\mathcal{V}$ in $\mathcal{H}$ the problems of describing $\overline{\mathbf{T}}_{i}(n)$ and of determining the cardinality of $\mathbf{F}_{\mathcal{V}}(n)$ reduce to counting the number of elements in $E_{i}$ and $E_{\leq \ell}$.

For example, if $\mathcal{V}$ is the variety generated by $\mathbf{C}_{3}=\mathbf{H}(P)$ for $P$ the 3 -element chain, then the only subdirectly irreducible algebras in $\mathcal{V}$ are $\mathbf{C}_{2}$ and $\mathbf{C}_{3}$. Among the valuations in $E_{\leq \ell}$ there are $2^{n-\ell}$ valuations $v$ for which $\mathbf{A} \lg (v)=\mathbf{C}_{2}$ and $3^{n-\ell}-2^{n-\ell}$ with $\mathbf{A} \lg (v)=\mathbf{C}_{3}$. So we have

$$
\overline{\mathbf{T}}_{\leq \ell}(n) \cong\left(\mathbf{C}_{2}\right)^{3^{n}} \text { and }\left|\mathbf{F}_{\mathcal{V}}(n)\right|=\sum_{\ell=1}^{n}(-1)^{\ell-1}\left(\begin{array}{l}
n \\
\ell
\end{array}\right) 2^{3^{n}} .
$$

This result may be found in [14].

We use Theorem 3.14 to determine the cardinality of the finitely generated free algebras for $\mathcal{V}=\mathcal{H}$. We first describe $E_{\leq \ell}$. Let $v \in E_{\leq \ell}$ with $\mathbf{A}=\mathbf{A} \lg (v)$. The subdirectly irreducible algebra $\mathbf{A}$, which is generated by the elements $e$ and the $v\left(x_{j}\right)$ for $\ell+1 \leq j \leq n$, has at most $n-\ell$ elements strictly below $e$. Let $R=\left\{x_{j} \in X: v\left(x_{j}\right)<e\right\}$ and $T=\left\{x_{j} \in X: v\left(x_{j}\right)=1\right\}$. We form a quasi-order $\sigma_{v}$ consisting of the union of these sets:

- $R \times(X-R)$,

- a quasi-order $\rho$ on $R$ given by $\rho=\left\{\left(x_{j}, x_{k}\right) \in R^{2}: v\left(x_{j}\right) \leq v\left(x_{k}\right)\right\}$,

- $X \times T$,

- the diagonal $\left\{\left(x_{j}, x_{j}\right): x_{j} \in X\right\}$.

If $|R|=r$, then the number of ways the set $R$ and the quasi-order $\rho$ on $R$ can be chosen is $\left(\begin{array}{c}n-\ell \\ r\end{array}\right) q_{r}$, where $0 \leq r \leq n-\ell$ and $q_{r}$ is the number of quasi-orders on an $r$-element set. Having chosen $r$ of the $v\left(x_{j}\right)$ to have value strictly less than $e$ there are at most $2^{n-\ell-r}$ ways to choose the set $T$. Hence

$$
\left|E_{\leq \ell}\right| \leq \sum_{r=0}^{n-\ell}\left(\begin{array}{c}
n-\ell \\
r
\end{array}\right) q_{r} 2^{n-\ell-r}
$$

This upper bound is actually obtained since for every set $R \subseteq\left\{x_{\ell+1}, \ldots, x_{n}\right\}$ of cardinality $r$ and every quasi-order $\rho$ defined on $R$, and for every choice of a set $T \subseteq\left\{x_{\ell+1}, \ldots, x_{n}\right\}-R$ 
with the quasi-order $\tau$ on $X$ given by

$$
\begin{aligned}
\tau= & \left\{\left(x_{j}, x_{k}\right): x_{j} \in X, x_{k} \in T\right\} \\
& \cup\left\{\left(x_{j}, x_{k}\right): x_{j} \in X, x_{k} \in T\right\} \\
& \cup\left\{\left(x_{j}, x_{j}\right): x_{j} \in X\right\}
\end{aligned}
$$

there is a subdirectly irreducible algebra $\mathbf{A}=\mathbf{H}(P)$ for $P$ the ordered set $X /(\rho \cup \tau)$. For this $\mathbf{A}$, the element 1 is $T /(\rho \cup \tau)$, the element $e$ is $(X-(R \cup T)) /(\rho \cup \tau)$, and each $x_{j} /(\rho \cup \tau)$ for $x_{j} \in R$ is strictly below $e$. If $v: X \rightarrow \mathbf{A}$ is defined by $v\left(x_{i}\right)=x_{i} /(\rho \cup \tau)$, then $v(X)$ generates $\mathbf{A}, v \in E_{\leq \ell}$, and $\sigma_{v}=\rho \cup \tau$. Thus,

$$
\left|E_{\leq \ell}\right|=\sum_{r=0}^{n-\ell}\left(\begin{array}{c}
n-\ell \\
r
\end{array}\right) q_{r} 2^{n-\ell-r} .
$$

An application of Theorem 3.14 gives the following result from [3].

\subsection{Theorem}

$$
\left|\mathrm{F}_{\mathcal{H}}(n)\right|=\sum_{\ell=1}^{n}(-1)^{\ell-1}\left(\begin{array}{l}
n \\
\ell
\end{array}\right) 2^{\sum_{r=0}^{n-\ell}\left(\begin{array}{c}
n-\ell \\
r
\end{array}\right) q_{r} 2^{n-\ell-r}} .
$$

The values of $\left|\mathrm{F}_{\mathcal{H}}(n)\right|$ for $n=1,2$, and 3 are 2,14 , and 12266 respectively.

\section{Structure via direct product decomposition}

In this section we describe a wide class of varieties for which the finitely generated free algebras have a direct product decomposition into directly indecomposable algebras where the structure of these indecomposables can be described in terms of free algebras of associated varieties and where the multiplicity of each indecomposable in the product is linked in a strong way to free algebras in another associated variety.

4.1 Definition A finite nontrivial algebra $\mathbf{Q}$ is quasiprimal if every nontrival subalgebra of $\mathbf{Q}$ is simple and the variety generated by $\mathbf{Q}$ is congruence permutable and congruence distributive.

There is an extensive literature on quasiprimal algebras and the varieties that they generated. We mention [20] and [16] as important sources of information about quasiprimal algebras and the varieties they generate.

Let $\mathbf{Q}$ be a quasiprimal algebra and $\mathcal{Q}$ the variety that it generates. It is known that the variety $\mathcal{Q}$ is semisimple, that is, every subdirectly irreducible algebra in $\mathcal{Q}$ is a simple algebra. Moreover, every simple algebra in $\mathcal{Q}$ is a subalgebra of $\mathbf{Q}$. If $\mathbf{A}$ is any finite algebra in $\mathcal{Q}$, then from the congruence distributivity of $\mathcal{Q}$ it follows that the congruence lattice Con $\mathbf{A}$ is a distributive lattice in which each element is a meet of coatoms, and therefore is a finite Boolean lattice. From the congruence permutability of $\mathcal{Q}$ we have that if $\alpha$ and $\beta$ are complements in this lattice, then $\mathbf{A}$ is isomorphic to $\mathbf{A} / \alpha \times \mathbf{A} / \beta$. If $\alpha_{1}, \ldots, \alpha_{r}$ are the coatoms of Con $\mathbf{A}$, then $\mathbf{A} \cong \mathbf{A} / \alpha_{1} \times \cdots \times \mathbf{A} / \alpha_{r}$ and each $\mathbf{A} / \alpha_{i}$ is a subalgebra of $\mathbf{Q}$. So 
every finite algebra in $\mathcal{Q}$ is a direct product, in a unique way, of simple algebras. In particular for every positive integer $n$ we may write

$$
\mathbf{F}_{\mathcal{Q}}(n)=\prod_{j=1}^{r} \mathbf{B}_{j}
$$

where each $\mathbf{B}_{j}$ is a simple subalgebra of $\mathbf{Q}$ and the kernel of the projection $p r_{j}$ onto $\mathbf{B}_{j}$ is a coatom, say $\alpha_{j}$, of the congruence lattice of $\mathbf{F}_{\mathcal{Q}}(n)$.

We rephrase (4.1) in terms of $\mathbf{G e}(X, R)$ for $X=\left\{x_{1}, \ldots, x_{n}\right\}$ and $R$ a set of valuations to subalgebras of $\mathbf{Q}$. For $x_{i} \in X$ let $\bar{x}_{i}$ be the $r$-tuple $\left(\bar{x}_{i}(1), \ldots, \bar{x}_{i}(r)\right)$ in (4.1). For each $1 \leq j \leq r$ let $v_{j}$ be the function from $X$ to $B_{j}$ for which $v_{j}\left(x_{i}\right)=\bar{x}_{i}(j)$. Then $v$ is a valuation to $\mathbf{B}_{j}$. If $R=\left\{v_{1}, \ldots, v_{r}\right\}$, then the array corresponding to $\mathbf{G e}(X, R)$ is identical to $\prod_{j=1}^{r} \mathbf{B}_{j}$ in (4.1). On the other hand, let $v \in \mathbf{Q}^{X}$ be arbitrary. If $\mathbf{B}$ is the subalgebra of $\mathbf{Q}$ generated by $v(X)$, then $v$ is a valuation to $\mathbf{B}$, the algebra $\mathbf{B}$ is simple, and the kernel of the homomorphic extension of $v$ to all of $\mathbf{F}_{\mathcal{Q}}(n)$ is a coatom of $\mathbf{C o n}\left(\mathbf{F}_{\mathcal{Q}}(n)\right)$. So there is a $1 \leq j \leq r$ and an isomorphism $h: \mathbf{B} \rightarrow \mathbf{B}_{j}$ such that $h\left(v\left(x_{i}\right)\right)=\bar{x}_{i}(j)=v_{j}\left(x_{i}\right)$. Thus $v$ and $v_{j}$ are equivalent in the sense of Definition 1.5. In fact, if $v, v^{\prime} \in Q^{X}$ are valuations that are equivalent in the sense of Definition 1.5, then the homomorphisms $h$ and $h^{\prime}$ between $\mathbf{A} \lg (v)$ and $\mathbf{A} \lg \left(\mathrm{v}^{\prime}\right)$ for which $v^{\prime}=h v$ and $v=h^{\prime} v^{\prime}$ must be isomorphisms since every subalgebra of $\mathbf{Q}$ is simple. So the transversal of valuations of Definition 1.5 can be chosen to be the set $R=\left\{v_{1}, \ldots, v_{r}\right\}$.

Therefore, the structure of $\mathbf{F}_{\mathcal{Q}}(n)$ is determined by a transversal, say $T$, of the pairwise nonisomorphic subalgebras of $\mathbf{Q}$ and for each algebra $\mathbf{B}$ in $T$, the number, say $m(\mathbf{B})$, of factors in the representation in (4.1) that are isomorphic to $\mathbf{B}$. The value of $m(\mathbf{B})$ is completely determined by the subuniverses and the automorphisms of B. Namely, consider the set of all $v \in \mathbf{B}^{X}$ for which $v(X)$ is not a subset of any proper subuniverse of $\mathbf{B}$, and for this set of valuations to $\mathbf{Q}$, choose a subset whose elements are pairwise inequivalent with respect to the equivalence relation of Definition 1.5. This pairwise inequivalence can be determined by only using automorphisms of $\mathbf{B}$ since $\mathbf{B}$ is a simple algebra. From $T$ and the values of $m(\mathbf{B})$ as $\mathbf{B}$ ranges over $T$ we have the direct product decomposition of $\mathbf{F}_{\mathcal{Q}}(n)$ given in (4.1). Note that in the case that the algebra $\mathbf{B}$ is rigid (i.e., no proper automorphisms), then $m(B)$ is just the number of $v \in \mathbf{B}^{X}$ for which $v(X)$ is not contained in any proper subuniverse of $\mathbf{B}$.

So by the analysis given in the previous paragraphs, the structure of finitely generated free algebras in a variety $\mathcal{Q}$ generated by a quasiprimal algebra $\mathbf{Q}$ can be described. Each is of the form $\mathbf{G e}(X, R)$ for $R$ a set of valuations that is free for $\mathcal{Q}$ and independent in the sense of Definition 1.2. The set $R$ of valuations can, in principle, be determined from the collection of subuniverses and automorphisms of $\mathbf{Q}$.

In the remainder of this section we obtain a structure theorem for free algebras that builds on the quasiprimal construction just given. The full theory is given in [2]. The presentation that we now give makes use of the $\mathbf{G e}(X, R)$ construction. We first present a detailed illustrative example.

4.2 Example Stone algebras: The variety $\mathcal{V}$ of Stone algebras is the subvariety of the variety of all distributive pseudocomplemented lattices that satisfy the identity $x^{*} \vee x^{* *}=1$. The only nontrivial subvariety of Stone algebras is the variety $\mathcal{B}$ of Boolean algebras. The $3-$ element Stone algebra $\mathbf{S}=\left\langle\{0,1,2\}, \wedge, \vee,{ }^{*}, 0,1\right\rangle$ has the lattice structure of the 3 -element chain $0<2<1$ with the pseudocomplement operation ${ }^{*}$ given by $0^{*}=1,1^{*}=0$, and 
$2^{*}=0$. It is known that the variety $\mathcal{V}$ of Stone algebras is generated by $\mathbf{S}$. Stone algebras have been thoroughly studied during the past 50 years. The structure of free Stone algebras was presented by R. Balbes and A. Horn in [1].

The only subdirectly irreducible Stone algebras are $\mathbf{S}$ and the 2-element Boolean algebra $\mathbf{B}=\left\langle\{0,1\}, \wedge, \vee,{ }^{*}, 0,1\right\rangle$. Let $h$ denote the homomorphism from $\mathbf{S}$ to $\mathbf{B}$ given by $h(0)=0$ and $h(1)=h(2)=1$. If $\alpha$ is the kernel of $h$, then $\alpha$ is a coatom of the congruence lattice of $\mathbf{S}$, the congruence lattice of $\mathbf{S}$ is $0<\alpha<1$ with $\alpha$ having $\{1,2\}$ as its only nontrivial block.

The free algebra $\mathbf{F}_{\mathcal{V}}(n)$ is a subdirect product of copies of $\mathbf{S}$ and $\mathbf{B}$. Let $U$ be the set of all valuations of $X$ to $\mathbf{S}$ or $\mathbf{B}$. There are $2^{n}$ valuations to $\mathbf{B}$ and $3^{n}-2^{n}$ valuations to $\mathbf{S}$ since every valuation to $\mathbf{S}$ must have 2 in its range. So $\mathbf{F}_{\mathcal{V}}(n)$ can be represented as the array $\mathbf{G e}(X, U)$. We define an equivalence relation $\sim_{\mathbf{B}}$ on $U$ by $v \sim_{\mathbf{B}} w$ if and only if $h\left(v\left(x_{i}\right)\right)=h\left(w\left(x_{i}\right)\right)$ for all $x_{i} \in X$. Clearly, there are $2^{n}$ equivalence classes for $\sim_{\mathbf{B}}$. If $Z \subset U$ is an equivalence class of $\sim_{\mathbf{B}}$ we wish to describe the projection of $\operatorname{Ge}(X, U)$ on $Z$. That is, we wish to describe $\mathbf{G e}(X, Z)$. We compute an illustrative example with the UAC program to see what is going on.

So let $n=4$ and suppose $Z$ consists of those valuations $v$ for which $h v\left(x_{1}\right)=h v\left(x_{2}\right)=$ $h v\left(x_{3}\right)=1$ and $h v\left(x_{4}\right)=0$. Then $v\left(x_{4}\right)=0$ but $v\left(x_{i}\right) \in\{1,2\}$ for $1 \leq i \leq 3$. So $|Z|=8$. The algebra $\operatorname{Ge}(X, Z)$ is generated by $\bar{x}_{1}, \ldots, \bar{x}_{4}$. Table 4 contains the output of the UAC program's computation of the subalgebra of $\mathbf{S}^{3}$ generated by $\bar{x}_{1}, \bar{x}_{2}, \bar{x}_{3}$ and $\bar{x}_{4}$.

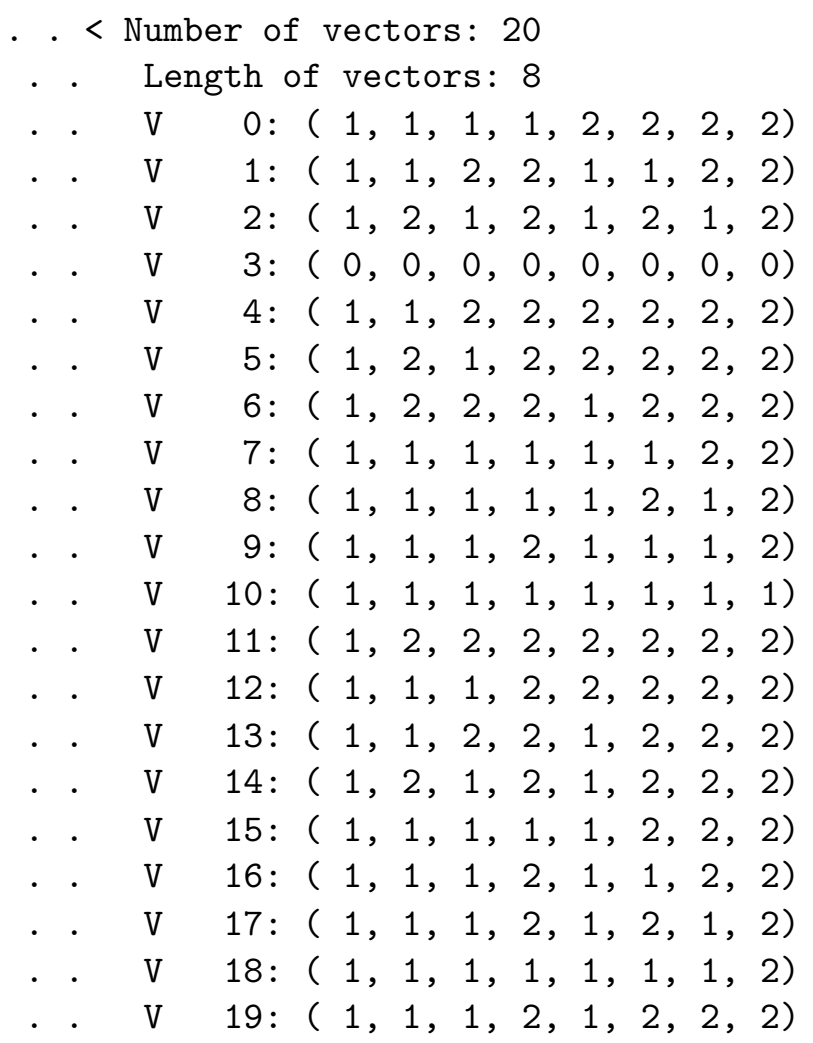

Table 4

The generators $\bar{x}_{1}, \bar{x}_{2}, \bar{x}_{3}$ and $\bar{x}_{4}$ of $\mathbf{G e}(X, Z)$ in Table 4 are labelled V0, V1, V2, and $\mathrm{V} 3$ respectively. The elements of each row of $\mathbf{G e}(X, Z)$ are all in the same $\alpha$ class, that is, 
each row is constant with respect to the homomorphism $h$. Of the 20 rows in $\mathbf{G e}(X, Z), 19$ lie in $\{1,2\}^{8}$ and one is in $\{0\}^{8}$. The operations $\wedge$ and $\vee$, when restricted to $\{1,2\}$ behave as lattice operations on the distributive lattice $\mathbf{D}$ with $2<1$. The 8 valuations of $\left\{x_{1}, x_{2}, x_{3}\right\}$ to $\mathbf{D}$ are coded as the eight columns of $\mathbf{G e}(X, Z)$. So the rows generated by V0, V1, and V2 by means of $\wedge$ and $\vee$ give the 18 elements of the free distributive lattice on 3 free generators. The operation ${ }^{*}$ applied to $\{1,2\}$ gives $\{0\}$, but the term ${ }^{* *}$ sends $\{1,2\}$ to $\{1\}$. So the row consisting of all 1's also appears. This accounts for the 19 rows whose elements are in the class $1 / \alpha$. There can only be one row whose elements are in the singleton class $0 / \alpha$. So all elements are accounted for. $\operatorname{So} \mathbf{G e}(X, Z)$ is isomorphic to $1 \oplus \mathbf{F}_{\mathcal{D}_{1}}(3)$, where $\mathcal{D}_{1}$ is the variety of upper bounded distributive lattices.

More generally, for arbitrary $n$, if $Z$ is an equivalence class of $\sim_{\mathbf{B}}$ and $n_{1}$ is the number of the elements $\bar{x}_{i}$ in $\mathbf{G e}(X, Z)$ that have $v\left(x_{i}\right) \in 1 / \alpha$ for all $v \in Z$, then as in the example the algebra $\mathbf{G e}(X, U)$ is isomorphic to $1 \oplus \mathbf{F}_{\mathcal{D}_{1}}\left(n_{1}\right)$. So the structure of $\mathbf{G e}(X, U)$ is quite clear. This algebra is also isomorphic to the free bounded distributive lattice on $n_{1}$ free generators, but we write it as an ordered sum in order to emphasize the origin of the two components of the algebra as determined by those $n_{1}$ variables that are mapped to 1 and those $n_{2}$ that are mapped to 0 . We shall see that this decomposition is an example of a more general one of the form $\mathbf{F}_{\mathcal{V}_{0}}\left(n_{0}\right) \cup \mathbf{F}_{\mathcal{V}_{1}}\left(n_{1}\right)$ for two varieties $\mathcal{V}_{0}$ and $\mathcal{V}_{1}$ with $n_{0}+n_{1}=n$

Next we consider how the different $\mathbf{G e}(X, Z)$ fit together. We view $\mathbf{F}_{\mathcal{S}}(n)$ as $\mathbf{G e}(X, U)$ for $U$ the set of all valuations to $\mathbf{S}$ and $\mathbf{B}$ and we view $\mathbf{F}_{\mathcal{B}}(n)$ as $\mathbf{G e}(X, W)$ with $W=\mathbf{B}^{X}$. Let $Z_{1}, \ldots, Z_{2^{n}}$ be a list of all the classes of $\sim_{\mathbf{B}}$. Let $g$ be the canonical onto homomorphism $g: \mathbf{F}_{\mathcal{S}}(n) \rightarrow \mathbf{F}_{\mathcal{B}}(n)$ that maps generators to generators. Since $g$ is onto, for each $\sim_{\mathbf{B}}$ class $Z$ there is a term $m_{Z}\left(x_{1}, \ldots, x_{n}\right)$ for which $v\left(g\left(m_{Z}\right)\right)=1$ if $v \in Z$ and $v\left(g\left(m_{Z}\right)\right)=0$ otherwise. Then for any $\bar{t} \in \mathbf{G e}(X, Z)$ the term $p_{Z, t}=t \wedge m_{Z}$ is such that $v\left(p_{Z, t}\right)=v(t)$ if $v \in Z$ and $v\left(p_{Z, t}\right)=0$ if $v \notin Z$. Let $\left(\bar{t}_{1}, \ldots, \bar{t}_{2^{n}}\right) \in \mathbf{G e}\left(X, Z_{1}\right) \times \cdots \times \mathbf{G e}\left(X, Z_{2^{n}}\right)$ be arbitrary. Form the term

$$
t\left(x_{1}, \ldots, x_{n}\right)=\bigvee_{1 \leq k \leq 2^{n}} p_{Z_{k}, t_{k}}
$$

For every $1 \leq k \leq n$ and every $v \in Z_{k}$ we have $v\left(t_{k}\right)=v(t)$. Therefore we conclude that $\mathbf{F}_{\mathcal{S}}(n) \cong \mathbf{G e}(X, U) \cong \mathbf{G e}\left(X, Z_{1}\right) \times \cdots \times \mathbf{G e}\left(X, Z_{2^{n}}\right)$.

By combining this with the characterization of $\mathbf{G e}(X, Z)$ given in the previous paragraph we get the result of [1]:

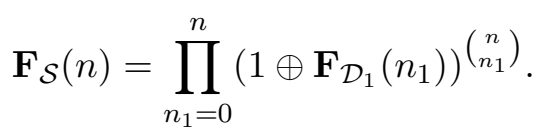

The paper [2] grew out of an attempt to understand a number of similar examples in the literature in which the free algebra $\mathbf{F}_{\mathcal{V}}(n)$ for a locally finite variety $\mathcal{V}$ has the form

$$
\mathbf{F}_{\mathcal{V}}(n)=\prod_{n_{0}+n_{1}=n}\left(\mathbf{F}_{\mathcal{V}_{0}}\left(n_{0}\right) \cup \mathbf{F}_{\mathcal{V}_{1}}\left(n_{1}\right)\right)^{\left(\begin{array}{c}
n \\
n_{1}
\end{array}\right)}
$$

or the more general form

$$
\mathbf{F}_{\mathcal{V}}(n)=\prod_{n_{1}+\cdots+n_{k}=n}\left(\mathbf{F}_{\mathcal{V}_{1}}\left(n_{1}\right) \cup \cdots \cup \mathbf{F}_{\mathcal{V}_{k}}\left(n_{k}\right)\right)^{\left(\begin{array}{c}
n \\
n_{1}, \ldots, n_{k}
\end{array}\right)}
$$


where the $\mathcal{V}_{i}$ are varieties derived from $\mathcal{V}$ in some way. The general phenomenon observed is that $\mathcal{V}$ is a locally finite variety and

$$
\mathbf{F}_{\mathcal{V}}(n)=\prod_{i} \mathbf{D}_{i}^{m_{i}}
$$

with each $\mathbf{D}_{i}$ a directly indecomposable factor of $\mathbf{F}_{\mathcal{V}}(n), m_{i}$ is the multiplicity of $\mathbf{D}_{i}$ in the product, each $\mathbf{D}_{i}$ is built from some algebras in some varieties associated with $\mathcal{V}$ and the values of the $m_{i}$ are determined by enumerating valuations of a particular form. The following is a description of some facts noticed in surveying these examples.

If $\mathcal{V}$ is one of the locally finite varieties examined, then the following facts were noted. The subvariety $\mathcal{V}_{0}$ generated by all finite simple algebras of $\mathcal{V}$ is generated by a quasiprimal algebra $\mathbf{Q}$. Typically $\mathbf{Q}$ is a familiar algebra such as the 2-element Boolean algebra $\mathbf{B}$. As we observed earlier, the free algebra $\mathbf{F}_{\mathcal{V}_{0}}(n)$ is of the form $\prod_{j=1}^{r} \mathbf{Q}_{j}$ with each $\mathbf{Q}_{j}$ a nontrivial subalgebra of $\mathbf{Q}$, and $r$ the cardinality of the set $R$ of valuations for which $\mathbf{F}_{\mathcal{V}_{0}}(n)$ is isomorphic to $\mathbf{G e}(X, R)$. As is the case for any variety generated by a quasiprimal algebra, the congruence lattice of $\mathbf{F}_{\mathcal{V}_{0}}(n)$ is a Boolean lattice with $r$ coatoms. If $\mathbf{F}_{\mathcal{V}}(n)$ is written as product of directly indecomposable algebras, then in the examples considered, the number of factors in this decomposition is also $r$, and $\mathbf{F}_{\mathcal{V}}(n)=\prod_{j=1}^{r} \mathbf{D}_{j}$ with each $\mathbf{D}_{j}$ directly indecomposable, the congruence lattice $\mathbf{C o n}\left(\mathbf{D}_{j}\right)$ has exactly one coatom, say $\alpha_{j}$, and $\mathbf{D}_{j} / \alpha_{i}$ is isomorphic to $\mathbf{Q}_{j}$. Moreover, in these examples, $\operatorname{Con}\left(\mathbf{F}_{\mathcal{V}}(n)\right) \cong \prod_{j=1}^{r} \operatorname{Con}\left(\mathbf{D}_{j}\right)$. Let $\mathcal{V}$ have similarity type $\tau$. For each $q \in Q$ let $\tau_{q}$ denote the similarity type consisting of all terms $t$ of type $\tau$ that satisfy $t^{\mathbf{Q}}(q, \ldots, q)=q$. We identify $\mathbf{F}_{\mathcal{V}}(n)$ with $\prod_{j=1}^{r} \mathbf{D}_{j}$ and let $p r_{j}: \mathbf{F}_{\mathcal{V}}(n) \rightarrow \mathbf{D}_{j}$ be the projection map and let $h_{j}: \mathbf{D}_{j} \rightarrow \mathbf{Q}_{j}$ be a homomorphism with kernel $\alpha_{j}$. For $1 \leq j \leq r$ and $q \in Q$ let $X_{j}^{q}=\left\{x_{i} \in X: h_{j}\left(p r_{j}\left(\bar{x}_{i}\right)\right)=q\right\}$. Let $\mathcal{V}_{q}$ denote the variety of similarity type $\tau_{q}$ that is generated by all algebras $\mathbf{A}_{j}^{q}$ whose universe is $h_{j}^{-1}(q) \subseteq D_{j}$ and whose operations are those term operations $t$ of $\mathbf{D}_{j}$ for which $t^{\mathbf{Q}}(q, \ldots, q)=q$. Then each $\mathbf{D}_{j}$ is built from subsets having the structure of $\mathbf{F}_{\mathcal{V}_{q}}\left(X_{j}^{q}\right)$ where $q$ ranges over the elements of $Q_{j}$. In the more familiar examples we have $D_{j}=\bigcup_{q \in Q_{j}} \mathbf{F}_{\mathcal{V}_{q}}\left(X_{j}^{q}\right)$.

For example, let us reconsider Example 4.2 of Stone algebras. Here the only simple algebra is the 2-element Boolean algebra $\mathbf{B}$ so the variety $\mathcal{S}_{0}$ is the variety $\mathcal{B}$ of Boolean algebras. The free algebra $\mathbf{F}_{\mathcal{B}}(n)$ is $\mathbf{B}^{2^{n}}$ since every valuation to $\mathbf{B}$ is needed to separate the elements of $\mathbf{F}_{\mathcal{B}}(n)$. So $r=2^{n}$ and $\operatorname{Con}\left(\mathbf{F}_{\mathcal{B}}(n)\right)$ is a Boolean algebra with $r$ coatoms. In the decomposition of $\mathbf{F}_{\mathcal{S}}(n)$ into directly indecomposables, $\prod_{j=1}^{r} \mathbf{D}_{j}$, the unique coatom of the congruence lattice of $\mathbf{D}_{j}$ is the kernel of the homomorphism $h_{j}$ onto $\mathbf{B}$ in which $h_{j}^{-1}(0)=\{0\}$ and $h_{j}^{-1}(1)=D_{j}-\{0\}$. The variety $\mathcal{S}$ is congruence distributive, so it follows from known results that for every $\mathbf{A} \in \mathcal{S}$, if $\mathbf{A}=\mathbf{A}_{1} \times \mathbf{A}_{2}$, then $\operatorname{Con} \mathbf{A} \cong \mathbf{C o n} \mathbf{A}_{1} \times \mathbf{C o n} \mathbf{A}_{2}$. The similarity types of $\tau_{0}$ and $\tau_{1}$ both include $\wedge, \vee$, ${ }^{* *}$; while $\tau_{0}$ includes the constant symbol 0 and $\tau_{1}$ contains the constant symbol 1. The algebras $\mathbf{A}_{j}^{0}$ for $1 \leq j \leq r$ are all 1-element algebras and so the variety $\mathcal{S}_{q}$ for $q=0$ is the trivial variety $\mathcal{T}$. For $q=1$ the algebras $\mathbf{A}_{j}^{1}$ are term equivalent to distributive lattices with a top element 1 and the variety $\mathcal{S}_{q}$ for $q=1$ is $\mathcal{D}_{1}$. For every $j$, we have $X_{j}^{k}=\left\{x_{i}: h_{j}\left(p r_{j}\left(\bar{x}_{i}\right)\right)=k\right\}$ for $k=0,1$. Then $\mathbf{D}_{j}$ has as its universe $\mathrm{F}_{\mathcal{T}}\left(X_{j}^{0}\right) \cup \mathrm{F}_{\mathcal{D}_{1}}\left(X_{j}^{1}\right)$.

In [2] general conditions on a variety $\mathcal{V}$ are presented and are proved to be sufficient to force the well-behaved direct product decomposition of $\mathbf{F}_{\mathcal{V}}(n)$ into indecomposables described in the previous paragraphs. We now sketch these results. 
A variety $\mathcal{V}$ is said to have the Fraser-Horn Property (FHP) if there are no skew congruences on finite products, i.e., for all $\mathbf{A}_{1}, \mathbf{A}_{2} \in \mathcal{V}$, every $\theta \in \mathbf{C o n}\left(\mathbf{A}_{1} \times \mathbf{A}_{2}\right)$ is a product congruence $\theta_{1} \times \theta_{2}$ with $\theta_{k} \in \mathbf{C o n}\left(\mathbf{A}_{k}\right)$ for $k=1$, 2 . If $\mathcal{V}$ has FHP, then $\operatorname{Con}\left(\mathbf{A}_{1} \times \mathbf{A}_{2}\right) \cong$ Con $\mathbf{A}_{1} \times \mathbf{C o n} \mathbf{A}_{2}$ for all $\mathbf{A}_{1}$ and $\mathbf{A}_{2}$ in $\mathcal{V}$. Various conditions that imply FHP are known. If $\mathcal{V}$ is congruence distributive, then $\mathcal{V}$ has FHP. Fraser and Horn [10] give a Mal'cev condition equivalent to FHP and they derive as a special case the following:

$A$ variety $\mathcal{V}$ has FHP if there are binary terms + and $\cdot$ and elements 0 and 1 in $\mathrm{F}_{\mathcal{V}}(3)$ such that for all $z \in \mathrm{F}_{\mathcal{V}}(3)$, we have $z \cdot 1=z+0=0+z=z$ and $z \cdot 0=0$.

So, for example every variety of rings with unit has FHP.

A finite algebra $\mathbf{A}$ has the Apple Property (AP) if for all $\beta \in$ Con $\mathbf{A}$, if $\beta<1_{A}$ is a factor congruence with $\mathbf{A} / \beta$ directly indecomposable, then the interval lattice $\left[\beta, 1_{A}\right]$ in $\mathbf{C o n} \mathbf{A}$ has exactly one coatom. A variety $\mathcal{V}$ has AP if every finite algebra in $\mathbf{A}$ has AP. Equivalently, $\mathcal{V}$ has AP if every directly indecomposable finite algebra in $\mathcal{V}$ has a congruence lattice with exactly one coatom. For example, a local ring has AP.

If an algebra or a variety has both FHP and AP, then we say it has FHAP. In [6] locally finite varieties with FHAP are shown to have finitely generated free algebras with the rich direct product structure described earlier in this section.

For any locally finite variety $\mathcal{V}$ let $\mathcal{V}_{0}$ be the subvariety generated by all finite simple algebras in $\mathcal{V}$. We call $\mathcal{V}_{0}$ the prime variety of $\mathcal{V}$. For arbitrary $\mathcal{V}$ little can be said about $\mathcal{V}_{0}$. However, in the event that a locally finite variety has FHAP and satisfies one additional condition, then the prime variety is especially well-behaved.

4.3 Theorem Let $\mathcal{V}$ be a locally finite variety with FHAP. Suppose every subalgebra of a finite simple algebra in $\mathcal{V}$ is a product of simple algebras. Then all of the following hold:

(1) $\mathcal{V}_{0}$ is congruence distributive.

(2) $\mathcal{V}_{0}$ is congruence permutable.

(3) Every finite member of $\mathcal{V}_{0}$ is a product of simple algebras.

(4) For every $n$ the free algebras $\mathbf{F}_{\mathcal{V}}(n)$ and $\mathbf{F}_{\mathcal{V}_{0}}(n)$ have the same number of directly indecomposable factors, and if $r$ denotes this number, then it is possible to write

$$
\mathbf{F}_{\mathcal{V}}(n)=\prod_{j=1}^{r} \mathbf{D}_{j} \text { and } \mathbf{F}_{\mathcal{V}_{0}}(n)=\prod_{j=1}^{r} \mathbf{Q}_{j}
$$

with the $\mathbf{D}_{j}$ and $\mathbf{Q}_{j}$ directly indecomposable, so that if $\alpha_{j}$ is the unique coatom of $\operatorname{Con}\left(\mathbf{D}_{j}\right)$, then $\mathbf{D}_{j} / \alpha_{j}$ and $\mathbf{Q}_{j}$ are isomorphic.

In the examples described in the earlier part of this section, the varieties satisfy the hypotheses of this theorem and the prime variety in each case is, in fact, a variety generated by a quasiprimal algebra.

4.4 Theorem Let $\mathcal{V}$ be a locally finite variety with FHAP. Suppose every subalgebra of a finite simple algebra in $\mathcal{V}$ is a product of simple algebras. Let $r, \mathbf{D}_{j}, \mathbf{Q}_{j}$ be as in Theorem 4.3 with $h_{j}: \mathbf{D}_{j} \rightarrow \mathbf{Q}_{j}$ a homomorphism with kernel $\alpha_{j}$. For $\mathbf{Q}$ a finite simple algebra in $\mathcal{V}$ and $q \in Q$, let $\tau_{q}$ be the similarity type of all terms $t$ for which $t^{\mathbf{Q}}(q, \ldots, q)=q$. For each $j$ 
with $\mathbf{Q}_{j} \cong \mathbf{Q}$, let $\mathbf{A}_{j}^{q}$ be the algebra with universe $h_{j}^{-1}(q) \subseteq D_{j}$ and operations those $t$ in $\tau_{q}$, and let $\mathcal{V}_{q}$ be the variety generated by all $\mathbf{A}_{j}^{q}$. If $X_{j}^{q}=\left\{x_{i} \in X: h_{j}\left(p r_{j}\left(\bar{x}_{i}\right)\right)=q\right\}$, then the algebra $\mathbf{A}_{j}^{q}$ contains a set that is the universe of an algebra isomorphic to $\mathbf{F}_{\mathcal{V}_{q}}\left(X_{j}^{q}\right)$.

The hypotheses of Theorems 4.3 and 4.4 are robust with many different varieties satisfying them. The examples alluded to earlier all satisfy them and thus Theorem 4.4 provides a uniform explanation of the description of the directly indecomposable direct factors of the finitely generated free algebras in those varieties.

In Theorem 4.4 we have that the universe of $\mathbf{D}_{j}$ is the disjoint union of the universes of the $\mathbf{A}_{j}^{q}$ as $q$ ranges over the elements of $\mathbf{Q}_{j}$ and that $\mathbf{A}_{j}^{q}$ contains a subalgebra isomorphic to $\mathbf{F}_{\mathcal{V}_{q}}\left(X_{j}^{q}\right)$. For varieties such as Stone algebras, $\mathbf{A}_{j}^{q}=\mathbf{F}_{\mathcal{V}_{q}}\left(X_{j}^{q}\right)$ and so $D_{j}=\bigcup_{q \in Q_{j}} \mathrm{~F}_{\mathcal{V}_{q}}\left(X_{j}^{q}\right)$. This situation represents a lower bound for the size of the $\mathbf{D}_{j}$. Moreover, it is possible to provide sufficient conditions on the behavior of the fundamental operations of $\mathcal{V}$ and $\mathcal{V}_{q}$ that guarantee that all the directly indecomposable $\mathbf{D}_{j}$ have this minimal structure.

On the other hand, it is possible for the size of $\mathbf{D}_{j}$ to exceed this lower bound. In [2] a condition is given that if added to the hypotheses of Theorem 4.4 forces $\mathbf{A}_{j}^{q} \cong \mathbf{F}_{\mathcal{V}_{q}}(X)$ for every $q \in Q_{j}$. Thus, in this case $D_{j}$ is the disjoint union of $\left|Q_{j}\right|$ sets, each corresponding to the universe of $\mathbf{F}_{\mathcal{V}_{q}}(X)$. Roughly speaking, this condition given in [2] is that for every $q, q^{\prime} \in Q_{j}$ there is a unary term $u_{q q^{\prime}}$ that maps $A_{j}^{q}$ onto $A_{j}^{q^{\prime}}$. Note that if this condition holds, then $\left|D_{j}\right|=\left|Q_{j}\right| \cdot\left|\mathrm{F}_{\mathcal{V}_{0}}(n)\right|$. An example of a variety in which this occurs is the variety $\mathcal{V}$ of rings generated by $\mathbf{Z}_{p^{2}}$, for $p$ any prime. As observed earlier, a variety of rings with unit has FHP. The variety $\mathcal{V}$ is known to have AP and thus $\mathcal{V}$ has FHAP. The only simple ring in $\mathcal{V}$ is $\mathbf{Z}_{p}$ and so the prime variety $\mathcal{V}_{0}$ is the variety generated by $\mathbf{Z}_{p}$. So $\mathbf{F}_{\mathcal{V}_{0}}(n) \cong\left(\mathbf{Z}_{p}\right)^{p^{n}}$. Theorem 4.4 applies. The number of factors in the decomposition of $\mathbf{F}_{\mathcal{V}}(n)$ into directly indecomposables is the same as that for $\mathbf{F}_{\mathcal{V}_{0}}(n)$, which is $p^{n}$. For each $q \in Z_{p}$ it can be shown that the variety $\mathcal{V}_{q}$ is the variety generated by the $p$-element group in which all $p$ elements are constants. The free algebra on $n$ free generators for this variety is easily seen to have $p^{n+1}$ elements. For each $q, q^{\prime} \in Z_{p}$ the unary term $u(x)=\left(x+q^{\prime}-q\right) \bmod (p)$ can serve as $u_{q q^{\prime}}$. So for each directly indecomposable factor $\mathbf{D}_{j}$ of $\mathbf{F}_{\mathcal{V}}(n)$ we have $\left|D_{j}\right|=p\left|\mathrm{~F}_{\mathcal{V}_{0}}(n)\right|=p^{n+2}$. All the $\mathbf{D}_{j}$ are isomorphic and thus $\left|\mathbf{F}_{\mathcal{V}}(n)\right|=\left(p^{n+2}\right)^{p^{n}}$.

\section{References}

[1] R. Balbes and A. Horn, Stone lattices, Duke Math J. 37 (1970), 537-546.

[2] J. Berman and W. J. Blok, The Fraser-Horn and Apple properties, Trans. Amer. Math. Soc. 302 (1987), 427-465.

[3] J. Berman and W. J. Blok, Free Łukasiewicz and hoop residuation algebras, Studia Logica, to appear.

[4] J. Berman and W. J. Blok, Algebras defined from ordered sets and the varieties they generate, manuscript, 2003.

[5] J. Berman and P. Idziak, Counting finite algebras in the Post varieties, Internat. J. Algebra Comput. 10 (2000), 323-337. 
[6] J. Berman and B. Wolk, Free lattices in some small varieties, Algebra Universalis 10 (1980), 269-289.

[7] G. Birkhoff, On the structure of abstract algebras, Proc. Cambridge Philos. Soc. 31 (1935), 433-454.

[8] S. Burris and H. P. Sanappanavar, A Course in Universal Algebra, Springer-Verlag, New York, 1981.

[9] A. Diego, Sur les Algèbres d'Hilbert, Collection de Logique Mathématique, Sr. A, Fasc. XXI Gauthier-Villars, Paris; E. Nauwelaerts, Louvain 1966.

[10] G. A. Fraser and A. Horn, Congruence relations on direct products, Proc. Amer. Math. Soc. 26 (1970), 390-394.

[11] http://www.cs.elte.hu/ ewkiss/software/uaprog/uaprog.html

[12] G. Grätzer and A. Kisielewicz, A survey of some open problems on $p_{n}$-sequences and free spectra of algebras and varieties, in Universal Algebra and Quasigroup Theory, A. Romanowska and J.D.H. Smith (eds.), Heldermann Verlag, Berlin, 1992, pp. 57-88.

[13] J. A. Green and D. Rees, On semigroups in which $x^{r}=x$, Proc. Cambridge Phil. Soc. 58 (1952), 35-40.

[14] F. Guzmán and C. Lynch, Varieties of positive implicative BCK-algebras - subdirectly irreducible and free algebras, Math. Japon. 37 (1992), 27-32.

[15] D. Hobby and R. McKenzie, The Structure of Finite Algebras, Amer. Math. Soc., Providence, 1988.

[16] K. Kaarli and A. F. Pixley, Polynomial Completeness in Algebraic Systems Chapman \& Hall/CRC, Boca Raton, 2001.

[17] H. Lakser, R. Padmanabhan, C. Platt, Subdirect decomposition of Płonka sums, Duke Math. J. 39 (1972), 485-488.

[18] R. N. McKenzie, G. F. McNulty, W. F. Taylor, Algebras, Lattices, Varieties, Vol. 1, Wadsworth \& Brooks/Cole, Monterey, 1987.

[19] J. Płonka, On free algebras and algebraic decompositions of algebras from some equational classes defined by regular equations, Algebra Universalis 1 (1971/72), 261-264.

[20] R. W. Quackenbush, Structure theory for equational classes generated by quasi-primal algebras, Trans. Amer. Math. Soc. 187 (1974), 127-145.

[21] Á. Szendrei, Clones in Universal Algebra, Séminaire de Mathématiques Supérieures 99, Université de Montréal, 1986.

[22] R. Wille, Subdirecte Produkte vollständiger Verbände, J. Reine Angew. Math. 283/284 (1976), 53-70. 